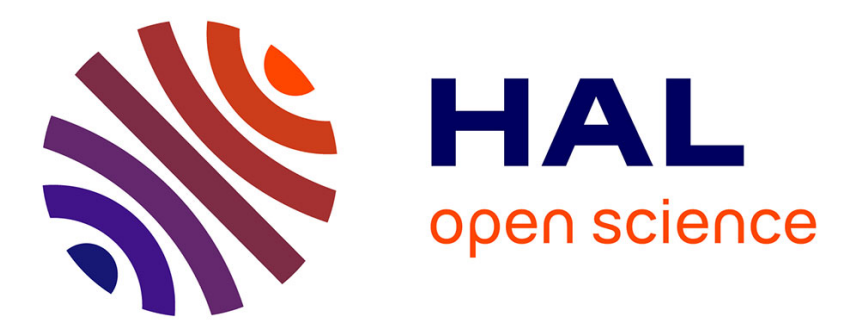

\title{
The Principles of d'Alembert, Jourdain, and Gauss in Nonsmooth Dynamics Part I: Scleronomic Multibody Systems
}

Christoph Glocker

\section{To cite this version:}

Christoph Glocker. The Principles of d'Alembert, Jourdain, and Gauss in Nonsmooth Dynamics Part I: Scleronomic Multibody Systems. Journal of Applied Mathematics and Mechanics / Zeitschrift für Angewandte Mathematik und Mechanik, 1998, 78 (1), pp.21 - 37. 10.1002/(SICI)15214001(199801)78:13.0.CO;2-W . hal-01379709

\section{HAL Id: hal-01379709 \\ https://hal.science/hal-01379709}

Submitted on 12 Oct 2016

HAL is a multi-disciplinary open access archive for the deposit and dissemination of scientific research documents, whether they are published or not. The documents may come from teaching and research institutions in France or abroad, or from public or private research centers.
L'archive ouverte pluridisciplinaire $\mathbf{H A L}$, est destinée au dépôt et à la diffusion de documents scientifiques de niveau recherche, publiés ou non, émanant des établissements d'enseignement et de recherche français ou étrangers, des laboratoires publics ou privés. 
Glocker, CH.

\title{
The Principles of d'Alembert, Jourdain, and Gauss in Nonsmooth Dynamics Part I: Scleronomic Multibody Systems
}

\begin{abstract}
Die vorliegende Arbeit behandelt die Berechnung der Beschleunigungen in starren Mehrkörpersystemen, wenn diese dem Einfluß mengenwertiger Kraftgesetze ausgesetzt sind. Die Kraftgesetze werden über nicht-glatte Potentialfunktionen berücksichtigt und über deren generalisiertes Differential dargestellt. Die dadurch entstehenden Punkt-Mengen-Abbildungen beinhalten neben glatten Kraftkennlinien auch Kräfte aus zweiseitigen Bindungen sowie Kombinationen aus beiden wie einseitige Bindungen, Trockenreibung oder vorgespannte Federn mit Spiel. Stöße werden nicht behandelt. Die klassischen Prinzipe von d'Alembert, Jourdain und Gauss werden mit Hilfe variationeller Ungleichungen verallgemeinert. Es wird ein streng konvexes Minimierungsproblem für die unbekannten Beschleunigungen des Systems aufgestellt, das in der klassischen Mechanik als das Prinzip des kleinsten Zwangs bekannt ist.
\end{abstract}

The paper treats the evaluation of the accelerations in rigid multibody systems which are subjected to set-valued force interactions. The interaction laws may be represented by non-smooth potential functions, and then derived through generalized differentiation. The resulting multifunctions contain the cases of smooth force characteristics, bilateral constraints, as well as combinations of them like unilateral constraints, dry friction, or prestressed springs with play. Impacts are excluded. A generalization of the classical principles of d'Alembert, Jourdain, and Gauss in terms of variational inequalities will be given. A strictly convex minimization problem depending on the unknown accelerations of the system will be stated, known in classical mechanics as the Principle of Least Constraints.

\section{Introduction}

In classical mechanics the concept of virtual work is used in order to classify displacement-dependent forces. Forces are called "active" or "applied forces" if they produce virtual work, otherwise they are called "passive" or "constraint forces". Both types are treated completely different with respect to formulation and evaluation of the equations of motion: Applied forces are continuous functions of the displacements and can therefore be directly evaluated when the displacements are known. Passive forces originate from bilateral constraints and can not be represented by such functions. Usually certain displacements are prescribed but the forces are arbitrary. The evaluation of the passive forces is thus done either by inverting the corresponding force characteristics which yields additional equations depending on the displacements known as constraints, or by choosing appropriate reduced sets of new coordinates, called "minimal coordinates", such that the constraints are automatically satisfied and thus eliminated from the equations of motion. This approach makes sense as long as the above mentioned classification applies to all forces within a multibody system. However, there are forces of intermediate type acting in some regions as applied forces, in other regions as constraints. Usually one becomes aware of them when dealing with dry friction, unilateral constraints, or impact effects. This type of forces leads to variational and hemivariational inequalities which are variational expressions for multivalued differential equations obtained by subdifferentiation or generalized differentiation of certain convex or nonconvex superpotential functions, as it is today well-known in continuum mechanics, cf. [1]-[3]. In classical mechanics only small attention is paid to the case of inequality constraints. One may find, for example, certain partial results on inequality Lagrange multipliers in the book [4], as well as in [5] and [6] where also the inequality form of the Principle of Virtual Work is discussed. Even in modern literature only a few works have appeared until now concerning inequality constraints and nondifferentiable energy functions. We refer especially to the papers [7]-[10] and to the few references given there.

Nearly all publications touching non-smooth dynamics are concentrated on the unilateral contact problem with Coulomb friction and/or impacts. They may be classified with respect to the level on which the interaction laws are stated: In [10] the displacement level is used in order to express the unilateral constraints which leads to the Principle of d'Alembert in inequality form. Every kind of dry friction as well as impacts are excluded because they demand a representation using velocities. Additionally the accelerations of the system are not accessible from this formulation. Based on the velocity level the complete friction-impact problem is treated in [8] as well as in [11], see e.g. [12] for further references. But even there only a few comments are made on how to determine the accelerations if they exist. In [13] - [17] the interaction laws are stated heuristically on the acceleration level after splitting off the impact equations. This approach has the advantage that accelerations may be directly computed when needed, but up to now an analytical back-up is missing. The aim of the presented paper is thus twofold. Besides of the main purpose of determining the accelerations of the dynamic system we try to find an analytical approach which connects the three different representations of interaction laws, i.e. the formulations using the displacement, velocity, and acceleration level, respec- 
tively. Secondly, we try to discuss how methods used in classical mechanics fit into this setting and how classical terminology might be understood when applied to non-smooth systems. We do not treat the impact itself. Impacts are beyond the scope of this paper. We are only interested in the accelerations of the system. Pre- and post-impact velocities, however, enter fully our description. Furthermore, we restrict ourselves to dry friction, i.e. Coulomb friction with given normal force. This restriction is necessary because all what follows is based on a potential theory approach. We allow, however, arbitrary friction laws like Stribeck-curves, and additionally multivalued displacement dependent interactions.

The paper is organized as follows. In Section 2 some preliminaries concerning differentiation of non-smooth functions are stated. It contains especially the definition of the generalized and the one-sided directional derivative together with the generalized gradient and the subdifferential which will be used throughout the rest of the paper. In Section 3 the equations of motion for non-smooth dynamic systems are derived from Lagrange's equations. Following [8], [18] the velocities are considered as functions of bounded variations, and the balance of the changes in momenta and forces is replaced by an equality of measures which covers both, impacts and impact free motion. This equality is then split into a purely atomic and a Lebesgue measurable part taking into account the impacts and the motion without velocity jumps which might be also nonsmooth, respectively. The remaining part of the paper treats only impact free motion. The impact equations are no longer considered. Section 4 deals with systems subjected to forces resulting from a displacement potential. Without making any difference between passive and active forces three variational inequalities are stated, together with the corresponding differential inclusions. Following classical mechanics they are called the Principles of d'Alembert, Jourdain, and Gauss, where the latter provides a strictly convex minimization problem closely related to the Principle of Least Constraints. In Section 5 a proof of the equivalence of the principles introduced above is sketched. The proof uses variational techniques and is mainly based on the classical idea that displacements and velocities must not be varied when velocity and acceleration variations are considered, respectively. Section 6 contains some remarks on how the reservoirs of forces provided by the three principles are connected together, and how to deal with accelerations when interested in the evolution of the dynamics leading into the past. In Sections $7-9$ the presented theory is applied to smooth interaction forces, unilateral, and bilateral constraints in order to show its compatibility with respect to the different classical approaches. Finally, in Section 10 the theory is extended to non-integrable potential functions depending on displacements, and also on velocities which makes it possible to include even forces related to viscous damping, dry friction, and nonholonomic constraints.

\section{Preliminaries}

In this section some basic definitions, notations, and properties of elements of nonsmooth analysis that will be used later in the paper are put together. Most of the material is taken from [1] and then applied to finite dimensional spaces. For detailed information we refer the reader to this book and especially to the references given there.

Let $D$ be a non-empty subset of $\mathbb{R}^{m}$ and $v \in D$. Following [19] the contingent cone $K_{D}$ and the tangent cone $T_{D}$ to $D$ at $v$ may be defined by

$$
\begin{aligned}
& K_{D}(v)=\left\{u \mid u \in \mathbb{R}^{m} ; \text { for } \mu_{n} \rightarrow 0_{+} \text {there exists } u_{n} \rightarrow u \text { with } v+\mu_{n} u_{n} \in D\right\}, \\
& T_{D}(v)=\left\{u \mid u \in \mathbb{R}^{m} ; \text { for } \mu_{n} \rightarrow 0_{+} \text {and } v_{n} \stackrel{D}{\rightarrow} v \text { there exists } u_{n} \rightarrow u \text { with } v_{n}+\mu_{n} u_{n} \in D\right\} .
\end{aligned}
$$

We set $K_{\emptyset}(v)=\emptyset, T_{\emptyset}(v)=\emptyset$, and $K_{D}(v)=\emptyset, T_{D}(v)=\emptyset$ if $v \notin D$. Both cones are closed, and, if not empty, they always contain 0 . Furthermore, $T_{D}(v)$ is convex and is always contained in $K_{D}(v), T_{D}(v) \subseteq K_{D}(v)$. For $D$ being convex we have $K_{D}(v)=T_{D}(v)$. If $D$ is a smooth manifold of class $C^{1}$, then $K_{D}(v)=T_{D}(v)$, and they are identified with the usual tangent vector space to $D$ at $v$ which is a subspace of $\mathbb{R}^{m}$. Note that for $v \in$ int $D$ both cones become $T_{D}(v)=K_{D}(v)=\mathbb{R}^{m}$. We say that a set $D$ is regular at a point $v$ if $T_{D}(v)=K_{D}(v)$. Regularity holds especially in the three cases mentioned above, i.e. when $D$ is a convex set, or $D$ is a smooth manifold, or $v$ is not a boundary point of $D$.

The normal cone $N_{D}(v)$ to $D$ at $v$ is defined as

$$
N_{D}(v)=\left\{w \mid w \in \mathbb{R}^{m},\langle u, w\rangle \leq 0 \text { for all } u \in T_{D}(v)\right\} .
$$

We set $N_{\emptyset}(v)=\emptyset$ and $N_{D}(v)=\emptyset$ if $v \notin D$. The normal cone is a closed, convex cone always containing 0 if not empty. In our case it is the polar (see e.g. [20]) of the closed, convex tangential cone, i.e. $N_{D}(v)$ and $T_{D}(v)$ are mutually polar. Observe that $N_{D}(v)=\mathbb{R}^{m} \Leftrightarrow T_{D}(v)=0$ and vice versa. If $T_{D}(v)$ is a halfspace then $N_{D}(v)$ degenerates to a ray perpendicular to the boundary of $T_{D}(v)$. If $T_{D}(v)$ is a subspace, for example the tangent space to a smooth manifold $D$, then $N_{D}(v)$ is its orthogonal complement.

The three cones introduced above will be applied in two different situations: By defining the generalized gradients of a function $f$ on $\mathbb{R}^{n}$ as subsets of $\mathbb{R}^{n+1}$ and, later in the paper, by investigating the properties of $f$ on its domain as subsets of $\mathbb{R}^{n}$. We will deal with functions taking values in the extended real line, i.e. $f: \mathbb{R}^{n} \rightarrow \mathbb{R} \cup\{+\infty\}=(-\infty,+\infty]$, but we will restrict ourselves to proper and lower semicontinuous (l.s.c.) functions only, i.e. functions for which $f \not \equiv+\infty$, and functions for which the set $\left\{x \mid x \in \mathbb{R}^{n}, f(x) \leq \lambda\right\}$ is closed for every $\lambda \in \mathbb{R}$ 
(see e.g. [1]). The effective domain of $f$ is defined by

$$
\operatorname{dom} f=\left\{x \mid x \in \mathbb{R}^{n}, f(x)<+\infty\right\},
$$

and the epigraph of $f$ by

$$
\text { epi } f=\left\{(x, \lambda) \mid \lambda \geq f(x), \lambda \in \mathbb{R}, x \in \mathbb{R}^{n}\right\},
$$

which is due to the properness and lower semicontinuity of $f$ a non-empty closed subset of $\mathbb{R}^{n+1}$ [19]. With the help of (2.3), (2.5) one defines the generalized gradient $\bar{\partial} f(x)$ to be (see e.g. [1])

$$
\bar{\partial} f(x)=\left\{y \mid y \in \mathbb{R}^{n},(y,-1) \in N_{\text {epi } f}(x, f(x))\right\},
$$

which is a convex, closed subset of $\mathbb{R}^{n}$ if $f(x)$ is finite. In [1] the generalized gradient (2.6) is used in order to define the generalized directional derivative $f^{\uparrow}(x, z)$ in the sense of Rockafellar,

$$
f^{\uparrow}(x, z)=\sup \{\langle z, y\rangle \mid y \in \bar{\partial} f(x)\}
$$

who has given another equivalent definition of it [21], [22]. Obviously one can express the generalized gradient $\bar{\partial} f(x)$ in terms of the generalized directional differential (2.7),

$$
\bar{\partial} f(x)=\left\{y \mid y \in \mathbb{R}^{n}, f^{\uparrow}(x, z) \geq\langle y, z\rangle \text { for all } z\right\},
$$

or by the hemivariational inequality

$$
y \in \bar{\partial} f(x) \Leftrightarrow f^{\uparrow}(x, z) \geq\langle y, z\rangle \text { for all } z \text {. }
$$

The generalized directional derivative $(2.7)$ as a function of $z$ has some important properties which will be used throughout the paper. As we will see in Section 3 this function provides the velocity and the acceleration potential corresponding to a given displacement potential of a dynamic system. Let $x$ be fixed in (2.7) and define

$$
g: z \rightarrow f^{\uparrow}(x, z)
$$

The following properties are proved in [22]: The function $g(z)$ is convex, l.s.c., positively homogeneous,

$$
g(\lambda z)=\lambda g(z) \text { for } \lambda>0,
$$

and subadditive,

$$
g(w+z) \leq g(w)+g(z),
$$

when $g(z)>-\infty$ for all $z$, and one has

$$
\text { epi } g=T_{\text {epi } f}(x, f(x)) \text {. }
$$

The function $g(z)$ may be considered as a "linearization of $f$ at $x$ as far as possible": The graph of $g$ consists of rays emanating from $(0,0)$ which are all tangent (in the sense of the definition $(2.2)$ ) to the set obtained by translating epi $f$ so that the point $(x, f(x))$ is moved to the origin. The cone generated by these rays is $T_{\text {epi } f}(x, f(x))$.

For convex functions $f$ one can show [1] that

$$
\bar{\partial} f(x)=\partial f(x) \quad \text { and } \quad f^{\uparrow}(x, z)=\liminf _{\bar{z} \rightarrow z} f^{\prime}(x, \bar{z}),
$$

where $\partial f(x)$ denotes the subdifferential from convex analysis [20] of $f$ at $x$ defined by

$$
\partial f(x)=\{y \mid f(z)-f(x) \geq\langle y, z-x\rangle \text { for all } z\},
$$

and $f^{\prime}(x, z)$ is the one-sided directional derivative for convex functions $f(x)$,

$$
f^{\prime}(x, z)=\inf _{\mu>0} \frac{1}{\mu}[f(x+\mu z)-f(x)]
$$

which exists for all $z$ when $f(x)$ is finite at $x$. Moreover,

$$
f^{\uparrow}(x, z) \leq f^{\prime}(x, z) \leq f(x+z)-f(x)
$$

where the first inequality follows from equation (2.14), and the second inequality is obvious when setting $\mu=1$ in (2.16).

For $f(x)$ continuously differentiable at $x$ we have

$$
\bar{\partial} f(x)=\nabla f(x) \quad \text { and } \quad f^{\uparrow}(x, z)=\langle\nabla f(x), z\rangle .
$$

Note that in this case the function $g(x)$ from $(2.10)$ can be replaced by the linear mapping $z \rightarrow\langle\nabla f(x), z\rangle$. Its graph is a hyperplane of $\mathbb{R}^{n+1}$ which is tangent to the graph of $f$ at the point $(x, f(x))$. 


\section{Lagrange's equations and nonsmooth dynamics}

We consider a mechanical system consisting of a finite number of rigid bodies subjected to some geometrical and physical interactions which are specified later. Such a system is called a multibody system (MBS). We assume the MBS to have $f$ degrees of freedom, due to some bilateral constraints, and we introduce the set of generalized coordinates $q \in \mathbb{R}^{f}$ in the classical sense. This set has the property that every possible (within the bilateral constraints) position and orientation of all of the bodies within the MBS can be uniquely determined by the values of $q$ and vice versa, and the dimension of $q$ is minimal.

Motion is defined by making $q$ dependent on time $t$. We are interested in a motion of the system on a compact time interval $I=\left[t_{\mathrm{A}}, t_{\mathrm{E}}\right]$. Following [8] we do not suppose the function $q: I \rightarrow \mathbb{R}^{f}$ differentiable everywhere. Instead we assume the velocities to be functions of bounded variations (BV) on $I$, i.e. we introduce a velocity function $u: I \rightarrow \mathbb{R}^{f}, u \in \mathrm{BV}\left(I, \mathbb{R}^{f}\right)$ such that

$$
\forall t \in I: \quad q(t)=q\left(t_{\mathrm{A}}\right)+\int_{t_{\mathrm{A}}}^{t} u(\tau) \mathrm{d} \tau,
$$

and hence $q$ is absolutely continuous on $I$ (see e.g. [23]). Since $u$ is of bounded variations its left limit $u^{-}(t)$ exists at every point of $\left(t_{\mathrm{A}}, t_{\mathrm{E}}\right]$, its right limit $u^{+}(t)$ exists at every point of $\left[t_{\mathrm{A}}, t_{\mathrm{E}}\right)$, and the set of points at which $u$ is discontinuous is at most countable and will be denoted by $\left\{t_{i}\right\}$. Moreover, we have $\dot{q}=u$ for Lebesgue-almost every $t \in\left[t_{\mathrm{A}}, t_{\mathrm{E}}\right]$, and $\dot{q}^{+}=u^{+}, \dot{q}^{-}=u^{-}$for every $t \in\left(t_{\mathrm{A}}, t_{\mathrm{E}}\right)$. Due to the discontinuities of $u$ the accelerations do not exist at times $t_{i}$, hence the derivative of $u$ must be understood in the distributional sense. Following [8] we introduce the differential measure of $u$ and denote it by $\mathrm{d} u$. With that measure one has, for every compact subinterval $\left[t_{k}, t_{l}\right]$ of $I$,

$$
\int_{\left[t_{k}, t_{l}\right]} \mathrm{d} u=u^{+}\left(t_{l}\right)-u^{-}\left(t_{k}\right),
$$

which admits a representation of the velocities in the form

$$
u^{+}(t)=u^{-}\left(t_{\mathrm{A}}\right)+\int_{\left[t_{\mathrm{A}}, t\right]} \mathrm{d} u, \quad u^{-}(t)=u^{-}\left(t_{\mathrm{A}}\right)+\int_{\left[t_{\mathrm{A}}, t\right)} \mathrm{d} u,
$$

when $t>t_{\mathrm{A}}$. Note especially that the term $u^{+}(t)-u^{-}(t)=\int_{[t]} \mathrm{d} u$ obtained by (3.2) when setting $t_{k}=t_{l}=t$ vanishes almost everywhere, except of the discontinuity points $t_{i}$ of $u$. It is known that the differential measure $\mathrm{d} u$ of a function $u \in \mathrm{BV}\left(I, \mathbb{R}^{f}\right)$ can be split into three parts, i.e.

$$
\mathrm{d} u=\mathrm{d} u_{\mathrm{L}}+\mathrm{d} u_{\mathrm{A}}+\mathrm{d} u_{\mathrm{C}} .
$$

The first term is absolutely continuous with respect to the 1-dimensional Lebesgue measure $\mathrm{d} t$ and has a density function which coincides almost everywhere with the derivative $\dot{u}$ of $u$,

$$
\mathrm{d} u_{\mathrm{L}}=\dot{u} \mathrm{~d} t .
$$

This means that $u \in \mathrm{BV}\left(I, \mathbb{R}^{f}\right)$ is differentiable a.e., $\dot{u} \in L^{1}\left(I, \mathbb{R}^{f}\right)$, and we denote the at most countable set of points at which $u$ is not differentiable by $\left\{t_{j}\right\}$. Note that the set of discontinuity points $\left\{t_{i}\right\}$ of $u$ and the set of points $\left\{t_{j}\right\}$ where $u$ is not differentiable may be different. Since $\dot{u}$ exists almost everywhere we may introduce its left limit $\dot{u}^{-}$and its right limit $\dot{u}^{+}$which are defined for every $t \in\left(t_{\mathrm{A}}, t_{\mathrm{E}}\right)$ with $\ddot{q}^{-}=\dot{u}^{-}$and $\ddot{q}^{+}=\dot{u}^{+}$. With it, equation (3.5) may be rewritten as

$$
\mathrm{d} u_{\mathrm{L}}=\dot{u}^{+} \mathrm{d} t, \quad \mathrm{~d} u_{\mathrm{L}}=\dot{u}^{-} \mathrm{d} t .
$$

Note that $\dot{u}^{+}=\dot{u}^{-}=\dot{u}$ for $[\mathrm{d} t]$-a.e. $t$, and hence $\dot{u}^{+} \mathrm{d} t=\dot{u}^{-} \mathrm{d} t=\dot{u} \mathrm{~d} t$ which guarantees the validity of (3.6). The second term in (3.4) is a 0 -dimensional measure, i.e. purely atomic and may be represented by

$$
\mathrm{d} u_{\mathrm{A}}=\left(u^{+}-u^{-}\right) \mathrm{d} \eta,
$$

where $\mathrm{d} \eta$ is concentrated on the set of discontinuities $\left\{t_{i}\right\}$ of $u$. It turns out that $\mathrm{d} \eta$ is the sum of the Dirac point measures $\mathrm{d} \delta_{i}$,

$$
\mathrm{d} \eta=\sum_{i} \mathrm{~d} \delta_{i}\left(I_{k l}\right) \quad \text { with } \quad \mathrm{d} \delta_{i}\left(I_{k l}\right)=\left\{\begin{array}{lll}
1 & \text { if } & t_{i} \in I_{k l} \\
0 & \text { if } & t_{i} \notin I_{k l}
\end{array},\right.
$$

where $I_{k l}$ denotes any one-dimensional cell, i.e. any open or closed or half-open interval with endpoints $t_{k}$ and $t_{l}$. The third term in (3.4), the measure $\mathrm{d} u_{\mathrm{C}}$, is singular with respect to $\mathrm{d} t$, and it may have support on sets which have Hausdorff dimensions between 0 and 1 , cf. [24]. We assume $\mathrm{d} u_{\mathrm{C}}=0$ because we are not dealing with MBS containing interaction forces of fractal type.

In order to derive the equations of motion of the MBS we will use Lagrange's equations of second kind [25] with $\dot{q}=u$ a.e.,

$$
\frac{\mathrm{d}}{\mathrm{d} t}\left(\frac{\partial T}{\partial u}\right)-\left(\frac{\partial T}{\partial q}\right)=f \quad \text { for }[\mathrm{d} t] \text {-a.e. } t \in I,
$$


where $f$ is the covariant vector of the generalized forces, and $T$ is the kinetic energy of the MBS which reads for the holonomic-scleronomic case

$$
T=\frac{1}{2} m_{i j} u^{i} u^{j}
$$

with $m_{i j}=m_{j i}$ depending on $q_{k}$. Same upper and lower indices have to be understood as implying summation. The evaluation of Lagrange's equations (3.9) yields the equilibrium of the changes in the linear and angular momenta and the forces of the MBS,

$$
m_{i j} \dot{u}^{j}+\Gamma_{i j k} u^{j} u^{k}=f_{i} \quad \text { for }[\mathrm{d} t] \text {-a.e. } t \in I .
$$

Here $\Gamma_{i j k}$ are the Christoffel symbols of first kind,

$$
\Gamma_{i j k}=\frac{1}{2}\left(m_{i k, j}+m_{i j, k}-m_{j k, i}\right)=\Gamma_{i k j},
$$

where $m_{a b, c}$ denotes the partial derivative of $m_{a b}$ with respect to $q_{c}, m_{a b, c}=\partial m_{a b} / \partial q_{c}$. Setting

$$
h_{i}=-\Gamma_{i j k} u^{j} u^{k}
$$

and $\left\{h_{i}\right\}=h,\left\{m_{i j}\right\}=M,\left\{\dot{u}^{i}\right\}=\dot{u},\left\{f_{i}\right\}=f$ we obtain from (3.11) the expression

$$
M(q) \dot{u}-h(q, u)=f \quad \text { for }[\mathrm{d} t] \text {-a.e. } t \in I
$$

with a symmetric and positive definite mass matrix $M \in \mathbb{R}^{f, f}$ (see e.g. [25]) and a vector $h \in \mathbb{R}^{f}$ which is a smooth function of $(q, u)$ containing the gyroscopical accelerations of the MBS.

Equation (3.14) is not suitable for further investigations because exactly the points of interest, i.e. the discontinuity points of $u$ and of its derivative $\dot{u}$ are excluded. Thus we try to get rid of the restriction "almost everywhere" and replace equation (3.14) as in [8] by the corresponding equality of measures,

$$
M(q) \mathrm{d} u-h(q, u) \mathrm{d} t=\mathrm{d} R,
$$

which holds for every $t \in I$. From the mechanical point of view this equality should be understood as an equilibrium of momenta and impulsions at the impact, and as a balance of changes in momenta and of forces for impact free motion. The measure $\mathrm{d} R$ in (3.15) contains both, Lebesgue-measurable forces $f$ and purely atomic impact impulsions $F$. Indeed, with

$$
\mathrm{d} R=f \mathrm{~d} t+F \mathrm{~d} \eta
$$

$\mathrm{d} u=\mathrm{d} u_{\mathrm{L}}+\mathrm{d} u_{\mathrm{A}}$ from (3.4), and $\mathrm{d} u_{\mathrm{A}}$ from (3.7), equation (3.15) becomes

$$
M(q) \mathrm{d} u_{\mathrm{L}}+M(q)\left(u^{+}-u^{-}\right) \mathrm{d} \eta-h(q, u) \mathrm{d} t=f \mathrm{~d} t+F \mathrm{~d} \eta
$$

and can be split into the atomic and the Lebesgue parts, i.e.

$$
M(q)\left(u^{+}-u^{-}\right) \mathrm{d} \eta=F \mathrm{~d} \eta, \quad M(q) \mathrm{d} u_{\mathrm{L}}-h(q, u) \mathrm{d} t=f \mathrm{~d} t .
$$

From the atomic part we obtain after evaluation of $\mathrm{d} \eta$ with respect to (3.8) the impact equations of the system,

$$
M\left(q_{i}\right)\left(u_{i}^{+}-u_{i}^{-}\right)=F_{i},
$$

where $q_{i}=q\left(t_{i}\right), u_{i}^{ \pm}=u^{ \pm}\left(t_{i}\right), F_{i}=F\left(t_{i}\right)$, and $t_{i}$ is any of the discontinuity points of the velocity $u$. Since the right equation in (3.18) is not affected by any changes of Lebesgue measure zero we may rewrite it with the help of (3.6) in order to obtain the two equivalent expressions

$$
M(q) \dot{u}^{+} \mathrm{d} t-h\left(q, u^{+}\right) \mathrm{d} t=f^{+} \mathrm{d} t, \quad M(q) \dot{u}^{-} \mathrm{d} t-h\left(q, u^{-}\right) \mathrm{d} t=f^{-} \mathrm{d} t .
$$

Note that the points where $u^{+} \neq u^{-}$and $\dot{u}^{+} \neq \dot{u}^{-}$are immaterial and hence Lebesgue-negligible. In this setting it is quite natural to assume similar properties for the forces $f$ in the right-hand sides of equations (3.18) and (3.20). Considered as functions of time we propose $f^{+}=f^{-}=f$ for $[\mathrm{d} t]$-a.e. $t$, where $f^{+}$and $f^{-}$denotes the right and left limits of $f$, respectively. Now we "divide" both equations in (3.20) by $\mathrm{d} t$. Considering $\ddot{q}^{ \pm}=\dot{u}^{ \pm}$and $\dot{q}^{ \pm}=u^{ \pm}$we obtain

$$
M(q) \ddot{q}^{+}-h\left(q, \dot{q}^{+}\right)=f^{+}, \quad M(q) \ddot{q}^{-}-h\left(q, \dot{q}^{-}\right)=f^{-},
$$

and have the following result: Every motion $q(t)$ which fulfills the impact equation (3.19) and one of the equations in (3.21), is also a solution of the measure equality (3.15). In the following we are only interested in the values of the accelerations. Thus the impact equation (3.19) will no longer be considered. The left equation in (3.21) represents the evolution of the MBS with respect to the future, because $\dot{q}^{+}$and $\ddot{q}^{+}$are the right limits of the velocities and accelerations, respectively. Suppose, for example, that $q, \dot{q}^{+}$, and $f^{+}$are given at a certain time point. In this case we may directly compute $\ddot{q}^{+}$from the left equation in (3.21) which describes the behaviour of the solution for succeeding times. Analogously, the right equation in (3.21) contains the evolution of the system pointing into the past, i.e. the evolution after reversal of the time arrow. 
Finally we want to discuss once again how the discontinuity points $\left\{t_{i}\right\}$ of the velocities $\dot{q}$ and $\left\{t_{j}\right\}$ of the accelerations $\ddot{q}$ are connected together. For almost every $t$ we have $\dot{q}^{+}=\dot{q}^{-}$and $\ddot{q}^{+}=\ddot{q}^{-}$, i.e. continuity. At the discontinuity points $\left\{t_{i}\right\}$ of the velocities we have $\dot{q}^{+} \neq \dot{q}^{-}$. These velocity jumps may be accompanied by continuous accelerations $\ddot{q}^{+}=\ddot{q}^{-}$or even discontinuous accelerations $\ddot{q}^{+} \neq \ddot{q}^{-}$as it can already be seen by the most primitive impact system: We consider the bouncing ball problem with completely elastic impact. At the impact time the velocity $\dot{q}^{-}<0$ is reversed, $\dot{q}^{+}=-\dot{q}^{-}$. The acceleration at the impact time is continuous because it is obtained by the equation of motion for the free-flight state $\ddot{q}=\ddot{q}^{+}=\ddot{q}^{-}=-g$, where $g$ denotes gravity. Now we consider the same system but under the influence of a completely inelastic impact. The acceleration before the impact is $\ddot{q}=-g$. At the impact the velocity $\dot{q}^{-}<0$ is changed to $\dot{q}^{+}=0$, i.e. after the impact the ball remains at the impact surface, and hence $\ddot{q}^{+}=0$. Suppose now that the velocities are continuous $\left(\dot{q}=\dot{q}^{+}=\dot{q}^{-}\right.$, i.e. $\left.t \notin\left\{t_{i}\right\}\right)$. Even in this case there might be an acceleration jump $\ddot{q}^{+} \neq \ddot{q}^{-}$as it can be seen by the following example: We consider a mass $m$ sliding on a plane with a velocity $\dot{q}>0$ under the influence of dry friction (here: Coulomb friction with a given normal load). The equations of motion during sliding are $\ddot{q}=-\mu g$, where $\mu$ is the coefficient of friction, and $g$ denotes gravity as above. The mass is decelerated until it comes to a rest. After it has stopped moving we have $\ddot{q}=0$, and hence for the transition point $\ddot{q}^{-}=-\mu g \neq 0=\ddot{q}^{+}$without any velocity jump.

\section{The Principles of d'Alembert, Jourdain, and Gauss: The holonomic case}

In this section we will define the Principles of d'Alembert, Jourdain, and Gauss for holonomic sceleronomic MBS, and we will show that the Principle of Gauss is equivalent to a strictly convex optimization problem which corresponds to the classical Principle of Least Constraints. In the following we are only interested in the right limits of the accelerations, i.e. in the values of $\ddot{q}^{+}$from the first equation in (3.21),

$$
M \ddot{q}^{+}-h=f^{+} .
$$

In order to obtain a complete description of the dynamics of the MBS we must introduce some force laws which connect the forces $f^{+}$, and the displacements $q$, and velocities $\dot{q}^{+}$. We will assume that these interaction laws may be derived from a potential function $V$ through generalized differentiation. We call the MBS to be holonomic if $V$ does not depend on the velocities $\dot{q}^{+}$, and we call it to be scleronomic if $V$ does not depend on time $t$. These definitions do not agree with the traditional where both terms are used only in context with constraints. However, we will see that constraints are now taken into account by the potential functions, thus these terms should be applied there. For the holonomic scleronomic case we thus have $V$ as a function of $q$, i.e. as a displacement potential, and we will equip it with the following properties: Let $V: \mathbb{R}^{f} \rightarrow \mathbb{R} \cup\{+\infty\}$ be a (non-smooth, non-convex) l.s.c. function with effective domain $C$ such that epi $V$ consists of regular points only, $T_{\text {epi } V}(q, V(q))=K_{\text {epi } V}(q, V(q))$. With that assumption we have excluded the so-called "reintrant corners" [26], the physical meaning of which being not yet well understood in multibody dynamics. The potentials $V(q)$ considered here thus "share the properties of nonsmooth convex and smooth nonconvex functions" rather than being general which, however, seems to be sufficient at the present time with respect to application problems. We will now assume force laws of the form

$$
-f^{+} \in \bar{\partial} V(q)
$$

where $\bar{\partial} V(q)$ denotes the generalized gradient (2.6) of $V$ at $q$. If we are interested in the accelerations from the past $\left(\ddot{q}^{-}\right)$we have to consider the second equation in (3.21) together with the force law $-f^{-} \in \bar{\partial} V(q)$. Note that both, $-f^{-}$ and $-f^{+}$belong to the same set $\bar{\partial} V(q)$ but might be different. They coincide, for example, if $\bar{\partial} V(q)$ consists of one element only, i.e. for example in the smooth case where we have $-f^{+}=-f^{-}=\nabla V(q)$.

By using the force law (4.2) we are able to take into account "smooth" interaction forces in the classical sense, for example springs with nonlinear characteristics, as well as unilateral and additional (this also means all) smooth bilateral constraints, and certain set-valued interactions. Examples will be given in Sections 7-9 and can also be found in [1]. Every kind of viscous and dry friction is excluded as well as nonholonomic constraints. They demand a representation via velocity potentials which is subject of Section 10 .

Combining (4.1) and (4.2) we get the differential inclusions of the system (see e.g. [10] for the convex case)

$$
-\left(M \ddot{q}^{+}-h\right) \in \bar{\partial} V(q) \text {. }
$$

With the help of (2.9) the inclusion (4.2) can also be expressed by means of a hemivariational inequality

$$
-f^{+} \in \bar{\partial} V(q) \Leftrightarrow V^{\uparrow}\left(q, q^{\star}-q\right) \geq\left\langle-f^{+}, q^{\star}-q\right\rangle, \quad q \in C, \quad \text { for all } q^{\star},
$$

where $V^{\uparrow}\left(q, q^{\star}-q\right)$ denotes the generalized directional derivative (2.7) of $V$ at $q$ in the direction $\left(q^{\star}-q\right)$. Together with (4.1) we obtain the expression

$$
-\left\langle M \ddot{q}^{+}-h, q^{\star}-q\right\rangle \leq V^{\uparrow}\left(q, q^{\star}-q\right) ; \quad q \in C, \quad \text { for all } q^{\star}
$$


which holds for every fixed time $t$, and which we will call the Principle of d'Alembert. Note that the variations $\left(q^{\star}-q\right)$ are not restricted to any subset of $\mathbb{R}^{f}$, thus values $V^{\uparrow}\left(q, q^{\star}-q\right)=+\infty$ which occur when $q$ is at the boundary of $C$ are allowed. The physical dimension of equation (4.5) is that of "work" [Nm]. Indeed, we will see in Section 9 when dealing with bilateral constraints that (4.5) leads to the classical result known as "constraint forces produce no work".

Like in classical mechanics we will now introduce a variational principle similar to equation (4.5) but with the physical dimension of a power $[\mathrm{Nm} / \mathrm{s}]$ which is also based on a potential function. For that purpose we define the function

$$
\Phi: p \rightarrow V^{\uparrow}(q, p)
$$

and call it the velocity potential of the MBS. Note that the velocity potential $\Phi$ is nothing else than the generalized directional derivative of $V$ at a point $q$ considered as a function of the direction $p$. By equations (2.10)-(2.13) the velocity potential is convex, l.s.c., positively homogeneous, and subadditive, and obviously $\operatorname{dom} \Phi=T_{C}(q)$ for every fixed $q$. We define the Principle of Jourdain for every fixed time $t$ to be

$$
\text { (J) } \quad-\left\langle M \ddot{q}^{+}-h, \dot{q}^{\diamond}-\dot{q}^{+}\right\rangle \leq \Phi^{\uparrow}\left(\dot{q}^{+}, \dot{q}^{\diamond}-\dot{q}^{+}\right) ; \quad q \in C, \quad \dot{q}^{+} \in T_{C}(q), \quad \text { for all } \dot{q}^{\diamond} .
$$

Although $\Phi$ is convex we have used the generalized directional derivative $\Phi^{\uparrow}\left(\dot{q}^{+}, \cdot\right)$ from $(2.7)$ but not the directional derivative $\Phi^{\prime}\left(\dot{q}^{+}, \cdot\right)$ from $(2.16)$. This is necessary in order to overcome difficulties at the boundary of $T_{C}(q)$ where both derivatives may not coincide, even for convex functions. The magnitude $\dot{q}^{\diamond}$ denotes just any arbitrary velocity in order to express that the inequality in equation (4.7) has to be valid for every direction $\left(\dot{q}^{\diamond}-\dot{q}^{+}\right)$. There is no connection between $q^{\star}$ in (4.5) and $\dot{q}^{\diamond}$ in (4.7), at least not now, and one must not understand $\dot{q}^{\diamond}$ to be the derivative of $q^{\star}$. Things will be clear in the next section where we will show how the principles (4.5) and (4.7) are connected together. With the help of equation (2.9) we may find analogously to (4.3) a differential inclusion which is equivalent to Jourdains Principle (4.7),

$$
-\left(M \ddot{q}^{+}-h\right) \in \partial \Phi\left(\dot{q}^{+}\right)
$$

where due to the convexity of $\Phi$ one may use the subdifferential (2.15) instead of the generalized gradient, cf. (2.14).

In order to define the Principle of Gauss we proceed in the same manner as above. We introduce the acceleration potential to be the generalized directional derivative of the velocity potential as a function of its direction,

$$
\Psi: p \rightarrow \Phi^{\uparrow}\left(\dot{q}^{+}, p\right)
$$

and notice that $\Psi(p)$ is also convex, l.s.c., positively homogeneous, and subadditive, and its effective domain is given by $\operatorname{dom} \Psi=T_{T_{C}(q)}\left(\dot{q}^{+}\right)$for every fixed $q, \dot{q}^{+}$. We define the Principle of Gauss for every fixed time $t$ to be

$$
\begin{aligned}
& -\left\langle M \ddot{q}^{+}-h, \ddot{q}^{\times}-\ddot{q}^{+}\right\rangle \leq \Psi^{\uparrow}\left(\ddot{q}^{+}, \ddot{q}^{\times}-\ddot{q}^{+}\right) ; \\
& q \in C, \quad \dot{q}^{+} \in T_{C}(q), \quad \ddot{q}^{+} \in T_{T_{C}(q)}\left(\dot{q}^{+}\right), \quad \text { for all } \ddot{q}^{\times},
\end{aligned}
$$

which has the physical dimension of a power per time $\left[\mathrm{Nm} / \mathrm{s}^{2}\right]$, and where the term $\ddot{q}^{\times}$has to be understood in the same sense as $\dot{q}^{\diamond}$ above. With the help of (2.9) we obtain the differential inclusion

$$
-\left(M \ddot{q}^{+}-h\right) \in \partial \Psi\left(\ddot{q}^{+}\right),
$$

which is an equivalent representation of (4.10).

Equation (4.11) constitutes the necessary and sufficient optimality conditions of a strictly convex optimization problem [20], which is obvious by rewriting it in the form

$$
0 \in M \ddot{q}^{+}-h+\partial \Psi\left(\ddot{q}^{+}\right) .
$$

The accelerations $\ddot{q}^{+}$are hence the optimal solutions of the strictly convex program

$$
\ddot{q}^{+}=\arg \min \left\{f\left(\ddot{\bar{q}}^{+}\right)\right\} ; \quad f\left(\ddot{q}^{+}\right)=\frac{1}{2}\left\langle M \ddot{q}^{+}, \ddot{q}^{+}\right\rangle-\left\langle h, \ddot{q}^{+}\right\rangle+\Psi\left(\ddot{q}^{+}\right),
$$

which is called in classical mechanics the Principle of Least Constraints. The cost function $f$ is strictly convex because $M$ is a symmetric and positive definite matrix, and the acceleration potential $\Psi$ is convex. Moreover, since $f$ is strictly convex, the optimal solutions $\ddot{q}^{+}$are unique.

\section{The connection between the principles}

In this section we will show that the three principles are equivalent, at least for a certain class of potential functions. For that purpose we have to consider the potential functions $V, \Phi$, and $\Psi$ at different time points which we will denote by lower indices for brevity. For example, $q_{n}$ means $q\left(t_{n}\right)$, and $q_{\dagger}$ means $q\left(t_{\dagger}\right)$ if we are interested in the displacements $q$ at time $t_{n}$ and time $t_{\dagger}$, respectively. In the first part of the section we will show the equivalence of d'Alembert's and Jourdain's principle, $(\mathrm{J})=(\mathrm{A})$. The connection between Jourdain's and Gauss' principle is discussed in the second part. As a result the equivalence $(G)=(J)$ is expected to hold for certain types of potential functions. 
The main idea of the proofs below consists of the following reflections (cf. [20], p. 235): Suppose that $t_{\dagger}$ is a given time point, and $q_{\dagger}$ and $\dot{q}_{\dagger}^{+}$are the displacements and right velocities at $t_{\dagger}$. Let $q_{1}, q_{2}, \ldots$ be a sequence of displacements at times $t_{1}>t_{2}>\ldots>t_{\dagger}$ which approaches $q_{\dagger}$ asymptotic to the half-line emanating from $q_{\dagger}$ in the direction of $\dot{q}_{\dagger}^{+}$. In this case, the forces $-f_{n}^{+} \in \bar{\partial} V\left(q_{n}\right)$ should tend toward the portion of the boundary of $\bar{\partial} V\left(q_{\dagger}\right)$ consisting of the forces $-f_{\dagger}^{+}$at which $\dot{q}_{\dagger}^{+}$is normal to $\bar{\partial} V\left(q_{\dagger}\right)$.

Let $t_{\dagger}$ be given, $t_{n}>t_{\dagger}$, and let $q_{\dagger}=q\left(t_{\dagger}\right), q_{n}=q\left(t_{n}\right)$ such that $q_{\dagger}, q_{n} \in \operatorname{dom} V=C$. Let $q_{n}^{\star}=q^{\star}\left(t_{n}\right)$ be any other displacement at time $t_{n}$ not necessarily belonging to dom $V$. In the following we restrict ourselves to displacements $q_{n}, q_{n}^{\star}$ sufficiently close to $q_{\dagger}$ and replace $V(q)$ by its first order approximation $\tilde{V}(q)$ at $q=q_{\dagger}$, i.e. we consider, instead of $V(q)$, the function

$$
\tilde{V}(q)=V\left(q_{\dagger}\right)+V^{\uparrow}\left(q_{\dagger}, q-q_{\dagger}\right) ; \quad \operatorname{dom} \tilde{V}=q_{\dagger}+T_{C}\left(q_{\dagger}\right),
$$

which is assumed to be proper. Note that $\tilde{V}(q)$ is a l.s.c. convex function because it is the translate of the l.s.c. positively homogeneous convex function $q \rightarrow V^{\uparrow}\left(q_{\dagger}, q\right)$ such that the point $(0,0)$ is moved to $\left(q_{\dagger}, V^{\uparrow}\left(q_{\dagger}, q_{\dagger}\right)\right)$. Note also that the effective domain of $\tilde{V}$ is a closed convex set, i.e. the set obtained by translating the origin of the closed convex cone $T_{C}\left(q_{\dagger}\right)$ to the point $q_{\dagger}$. Under these assumptions the following proposition (cf. [20], Theorem 24.6) holds.

Proposition 5.1: Let $\tilde{V}: \mathbb{R}^{f} \rightarrow(-\infty,+\infty]$ be the l.s.c. proper convex function defined in $(5.1)$ with $q_{\dagger} \in C$. Let $\left\{t_{n}\right\}$ be a sequence such that $t_{1}>t_{2}>\ldots>t_{\dagger}$, and $t_{n} \rightarrow t_{\dagger}$ for $n \rightarrow \infty$. Let $q(t)$ be an absolutely continuous function such that $q\left(t_{\dagger}\right)=q_{\dagger}, q\left(t_{n}\right)=q_{n}$ with $q_{n} \in \operatorname{dom} \tilde{V}$, and $q_{n}$ not necessarily distinct from $q_{\dagger}$. Then

$$
\begin{array}{lll}
\text { i) } & \tilde{\Phi}^{\uparrow}\left(\dot{q}_{\dagger}^{+}, \dot{\eta}_{\dagger}^{\diamond}\right) \leq \tilde{V}^{\uparrow}\left(q_{\dagger}, \dot{\eta}_{\dagger}^{\diamond}\right) & \text { for all } \dot{\eta}_{\dagger}^{\diamond}, \\
\text { ii) } & \limsup _{n \rightarrow \infty} \tilde{V}^{\uparrow}\left(q_{n}, \dot{\eta}_{\dagger}^{\diamond}\right) \leq \tilde{V}^{\uparrow}\left(q_{\dagger}, \dot{\eta}_{\dagger}^{\diamond}\right) & \text { for all } \dot{\eta}_{\dagger}^{\diamond}, \\
\text { iii) } & \limsup _{n \rightarrow \infty} \tilde{V}^{\uparrow}\left(q_{n}, \dot{\eta}_{\dagger}^{\diamond}\right) \leq \tilde{\Phi}^{\uparrow}\left(\dot{q}_{\dagger}^{+}, \dot{\eta}_{\dagger}^{\diamond}\right) & \text { for all } \dot{\eta}_{\dagger}^{\diamond},
\end{array}
$$

where $q_{n} \rightarrow q_{\dagger}$ as $n \rightarrow \infty, \tilde{\Phi}(\cdot)=\tilde{V}^{\uparrow}\left(q_{\dagger}, \cdot\right)$ is the velocity potential (4.6) of $\tilde{V}$ at $q_{\dagger}$, and $\dot{q}_{\dagger}^{+}$is the right velocity of $q$ at time $t_{\dagger}$ with $\dot{q}_{\dagger}^{+} \in T_{C}\left(q_{\dagger}\right)$.

Proof: By property (2.17), the one-sided generalized directional derivative $\tilde{\Phi}^{\uparrow}$ in (5.2) fulfills

$$
\tilde{\Phi}^{\uparrow}\left(\dot{q}_{\dagger}^{+}, \dot{\eta}_{\dagger}^{\diamond}\right) \leq \tilde{\Phi}\left(\dot{q}_{\dagger}^{+}+\dot{\eta}_{\dagger}^{\diamond}\right)-\tilde{\Phi}\left(\dot{q}_{\dagger}^{+}\right)
$$

Since $\tilde{\Phi}(\cdot)$ is subadditive $(2.12)$ we have

$$
\tilde{\Phi}\left(\dot{q}_{\dagger}^{+}+\dot{\eta}_{\dagger}^{\diamond}\right) \leq \tilde{\Phi}\left(\dot{q}_{\dagger}^{+}\right)+\tilde{\Phi}\left(\dot{\eta}_{\dagger}^{\diamond}\right)
$$

Combining equations (5.3) and (5.4) yields

$$
\tilde{\Phi}^{\uparrow}\left(\dot{q}_{\dagger}^{+}, \dot{\eta}_{\dagger}^{\diamond}\right) \leq \tilde{\Phi}\left(\dot{\eta}_{\dagger}^{\diamond}\right)
$$

which proves the first assertion, because $\tilde{\Phi}\left(\dot{\eta}_{\dagger}^{\diamond}\right)=\tilde{V}^{\uparrow}\left(q_{\dagger}, \dot{\eta}_{\dagger}^{\diamond}\right)$ by definition.

By assumption, $\tilde{V}(\cdot)$ is a proper convex function on $\mathbb{R}^{f}$, and $q_{n} \in \operatorname{dom} \tilde{V}$ with $\operatorname{dom} \tilde{V}$ closed. Thus, the function $\tilde{V}^{\uparrow}\left(\cdot, \dot{\eta}_{\dagger}^{\diamond}\right)$ is u.s.c. and assertion ii) holds.

In order to prove the third assertion we have to state the potential $\tilde{V}$ at different displacements corresponding to different time points. Let $q_{\dagger}=q\left(t_{\dagger}\right), q_{n}=q\left(t_{n}\right) \in \operatorname{dom} \tilde{V}$ as mentioned above, and let $q_{n}^{\star}=q^{\star}\left(t_{n}\right)$ be any other displacement, not necessarily belonging to $\operatorname{dom} \tilde{V}$. We have

$$
\tilde{V}^{\uparrow}\left(q_{n}, q_{n}^{\star}-q_{n}\right) \leq \tilde{V}\left(q_{n}^{\star}\right)-\tilde{V}\left(q_{n}\right)
$$

because $\tilde{V}(q)$ is convex and hence property (2.17) applies. Note that $q_{n}^{\star}$ may not belong to dom $\tilde{V}$, i.e. values $\tilde{V}\left(q_{n}^{\star}\right)=+\infty$ are allowed, but by assumption $q_{n} \in \operatorname{dom} \tilde{V}$, and hence $\tilde{V}\left(q_{n}\right)<+\infty$. Equation (5.6) holds for any arbitrary sequence $\left\{q_{n}^{\star}\right\}$. It holds particularly for sequences $\left\{q_{n}^{\diamond}\right\} \subset\left\{q_{n}^{\star}\right\}$ approaching $q_{\dagger}$ for $n \rightarrow \infty$. Thus we set

$$
q_{n}-q_{\dagger}=\tau_{n} \dot{q}_{n}^{+}, \quad q_{n}^{\diamond}-q_{\dagger}=\tau_{n} \dot{q}_{n}^{\diamond},
$$

where $\tau_{n}=t_{n}-t_{\dagger}>0$, and $\tau_{n} \downarrow 0, q_{n} \rightarrow q_{\dagger}, q_{n}^{\diamond} \rightarrow q_{\dagger}$ for $n \rightarrow \infty$. Note that

$$
\lim _{n \rightarrow \infty} \frac{1}{\tau_{n}}\left(q_{n}-q_{\dagger}\right)=\dot{q}_{\dagger}^{+}, \quad \lim _{n \rightarrow \infty} \frac{1}{\tau_{n}}\left(q_{n}^{\diamond}-q_{\dagger}\right)=\dot{q}_{\dagger}^{\diamond},
$$

which is the definition of the right derivatives of the absolutely continuous functions $q(t)$ and $q^{\diamond}(t)$ at time $t_{\dagger}$, where $q^{\diamond}(t)$ has been chosen such that $q^{\diamond}\left(t_{\dagger}\right)=q\left(t_{\dagger}\right)$ but $\dot{q}_{\dagger}^{\diamond}$ is arbitrary. This agrees with the well-known classical result that displacements must not be varied when velocity variations are considered. Since $q_{n} \in \operatorname{dom} \tilde{V}=q_{\dagger}+T_{C}\left(q_{\dagger}\right)$ by assumption (cf. also (5.1)), and $q_{n}-q_{\dagger}=\tau_{n} \dot{q}_{n}^{+}$by (5.7), and $\tau_{n}>0$ we have $\dot{q}_{n}^{+} \in T_{C}\left(q_{\dagger}\right)$ for every $n$ because $T_{C}\left(q_{\dagger}\right)$ is a closed convex cone. Especially for $n \rightarrow \infty$ we obtain $\dot{q}_{\dagger}^{+} \in T_{C}\left(q_{\dagger}\right)$. Putting (5.7) into (5.6) with $\left\{q_{n}^{\diamond}\right\} \subset\left\{q_{n}^{\star}\right\}$ yields

$$
\tilde{V}^{\uparrow}\left(q_{n}, \tau_{n}\left(\dot{q}_{n}^{\diamond}-\dot{q}_{n}^{+}\right)\right) \leq \tilde{V}\left(q_{\dagger}+\tau_{n} \dot{q}_{n}^{\diamond}\right)-\tilde{V}\left(q_{\dagger}+\tau_{n} \dot{q}_{n}^{+}\right) .
$$


With the help of (5.1) we evaluate the right-hand members of (5.9) and obtain

$$
\tilde{V}^{\uparrow}\left(q_{n}, \tau_{n}\left(\dot{q}_{n}^{\diamond}-\dot{q}_{n}^{+}\right)\right) \leq V^{\uparrow}\left(q_{\dagger}, \tau_{n} \dot{q}_{n}^{\diamond}\right)-V^{\uparrow}\left(q_{\dagger}, \tau_{n} \dot{q}_{n}^{+}\right) .
$$

Since $\tilde{V}^{\uparrow}\left(q_{n}, \cdot\right)$ and $V^{\uparrow}\left(q_{\dagger}, \cdot\right)$ are positively homogeneous and $\tau_{n}>0$, property (2.11) applies, and (5.10) becomes

$$
\tilde{V}^{\uparrow}\left(q_{n}, \dot{q}_{n}^{\diamond}-\dot{q}_{n}^{+}\right) \leq V^{\uparrow}\left(q_{\dagger}, \dot{q}_{n}^{\diamond}\right)-V^{\uparrow}\left(q_{\dagger}, \dot{q}_{n}^{+}\right) .
$$

Considering that $V^{\uparrow}\left(q_{\dagger}, \cdot\right) \equiv \tilde{V}^{\uparrow}\left(q_{\dagger}, \cdot\right)$ by equation $(5.1)$ and that $\tilde{V}^{\uparrow}\left(q_{\dagger}, \cdot\right) \equiv \tilde{\Phi}(\cdot)$ by definition we are now able to write the inequality (5.11) in the form

$$
\tilde{V}^{\uparrow}\left(q_{n}, \dot{q}_{n}^{\diamond}-\dot{q}_{n}^{+}\right) \leq \tilde{\Phi}\left(\dot{q}_{n}^{\diamond}\right)-\tilde{\Phi}\left(\dot{q}_{n}^{+}\right) .
$$

Equation (5.12) is valid for every sequence $\left\{\dot{q}_{n}^{\diamond}\right\}$. We choose sequences

$$
\dot{q}_{n}^{\diamond}-\dot{q}_{n}^{+}=\mu \dot{\eta}_{\dagger k}^{\diamond}, \quad \mu>0
$$

with arbitrary $\dot{\eta}_{\dagger k}^{\diamond}, \dot{\eta}_{\dagger}^{\diamond}$ such that $\dot{\eta}_{\dagger k}^{\diamond} \rightarrow \dot{\eta}_{\dagger}^{\diamond}$ when $k \rightarrow \infty$ and eliminate $\dot{q}_{n}^{\diamond}$ from (5.12), i.e.

$$
\tilde{V}^{\uparrow}\left(q_{n}, \dot{\eta}_{\uparrow k}^{\diamond}\right) \leq \frac{1}{\mu}\left[\tilde{\Phi}\left(\dot{q}_{n}^{+}+\mu \dot{\eta}_{\dagger k}^{\diamond}\right)-\tilde{\Phi}\left(\dot{q}_{n}^{+}\right)\right] .
$$

This inequality holds for any $\mu>0$, where the most restrictive case is given when the right-hand side attains its infimum with respect to $\mu$. In that case we have, together with the definition of the directional derivative (2.16),

$$
\tilde{V}^{\uparrow}\left(q_{n}, \dot{\eta}_{\dagger k}^{\diamond}\right) \leq \inf _{\mu>0} \frac{1}{\mu}\left[\tilde{\Phi}\left(\dot{q}_{n}^{+}+\mu \dot{\eta}_{\dagger k}^{\diamond}\right)-\tilde{\Phi}\left(\dot{q}_{n}^{+}\right)\right]=\tilde{\Phi}^{\prime}\left(\dot{q}_{n}^{+}, \dot{\eta}_{\dagger k}^{\diamond}\right)
$$

Now we let $k \rightarrow \infty$, i.e. $\dot{\eta}_{\dagger k}^{\diamond} \rightarrow \dot{\eta}_{\dagger}^{\diamond}$. Taking the "lim inf" of the left-hand member and the right-hand member of (5.15) we obtain

$$
\tilde{V}^{\uparrow}\left(q_{n}, \dot{\eta}_{\dagger}^{\diamond}\right) \leq \liminf _{\dot{\eta}_{\dagger k}^{\diamond} \rightarrow \dot{\eta}_{\dagger}^{\diamond}} \tilde{V}^{\uparrow}\left(q_{n}, \dot{\eta}_{\dagger k}^{\diamond}\right) \leq \liminf _{\dot{\eta}_{\dagger k}^{\diamond} \rightarrow \dot{\eta}_{\dagger}^{\diamond}} \tilde{\Phi}^{\prime}\left(\dot{q}_{n}^{+}, \dot{\eta}_{\dagger k}^{\diamond}\right)=\tilde{\Phi}^{\uparrow}\left(\dot{q}_{n}^{+}, \dot{\eta}_{\dagger}^{\diamond}\right)
$$

where the first inequality holds because $\tilde{V}^{\uparrow}\left(q_{n}, \cdot\right)$ is l.s.c., and the equality is due to (2.14). Finally we let $n \rightarrow \infty$, where $q_{n} \rightarrow q_{\dagger}$ and $\dot{q}_{n}^{+} \rightarrow \dot{q}_{\dagger}^{+}$by equations (5.7) and (5.8), respectively. Since $\tilde{V}^{\uparrow}\left(\cdot, \dot{\eta}_{\dagger}^{\diamond}\right)$ as well as $\tilde{\Phi}^{\uparrow}\left(\cdot, \dot{\eta}_{\dagger}^{\diamond}\right)$ are u.s.c. we may take the "lim sup" in order to obtain

$$
\limsup _{n \rightarrow \infty} \tilde{V}^{\uparrow}\left(q_{n}, \dot{\eta}_{\dagger}^{\diamond}\right) \leq \limsup _{n \rightarrow \infty} \tilde{\Phi}^{\uparrow}\left(\dot{q}_{n}^{+}, \dot{\eta}_{\dagger}^{\diamond}\right) \leq \tilde{\Phi}^{\uparrow}\left(\dot{q}_{\dagger}^{+}, \dot{\eta}_{\dagger}^{\diamond}\right) \quad \text { for all } \dot{\eta}_{\dagger}^{\diamond},
$$

which proves assertion iii). $\quad \|$

We expect that Proposition 5.1 applies also to $V(q)$ when taken from a certain class of non-convex functions. In this case we may write according to equation (5.2)

$$
\limsup _{n \rightarrow \infty} V^{\uparrow}\left(q_{n}, \dot{\eta}_{\dagger}^{\diamond}\right) \leq \Phi^{\uparrow}\left(\dot{q}_{\dagger}^{+}, \dot{\eta}_{\dagger}^{\diamond}\right) \leq V^{\uparrow}\left(q_{\dagger}, \dot{\eta}_{\dagger}^{\diamond}\right) \text { for all } \dot{\eta}_{\dagger}^{\diamond}
$$

This seems at least to be true when the graphs of $V$ and $\tilde{V}$ are locally $C^{1}$-diffeomorph at the point $\left(q_{\dagger}, V\left(q_{\dagger}\right)\right)$. A situation where (5.18) obviously does not hold is given when epi $V$ is not regular at $q_{\dagger}$, i.e. in the case of reintrant corners.

Theorem 5.2: The principles of d'Alembert (4.5) and Jourdain (4.7) are equivalent if (5.18) holds: Suppose that $q(t)$ is a given absolutely continuous function on the time interval $\left[t_{\mathrm{A}}, t_{\mathrm{E}}\right]$ such that d'Alembert's principle holds for every $t_{\dagger} \in\left[t_{\mathrm{A}}, t_{\mathrm{E}}\right]$. Then Jourdain's principle holds on $\left[t_{\mathrm{A}}, t_{\mathrm{E}}\right)$. If Jourdain's principle holds on $\left[t_{\mathrm{A}}, t_{\mathrm{E}}\right]$, then d'Alembert's principle also does.

Proof: We state d'Alembert's principle (4.5) at time $t_{n}>t_{\dagger}$ with $\left\{q_{n}^{\diamond}\right\} \subset\left\{q_{n}^{\star}\right\}$ and $q_{n}^{\diamond}-q_{n}=\tau_{n} \mu \dot{\eta}_{\dagger}^{\diamond}$ according to equations (5.7), (5.13),

$$
-\left\langle\left(M \ddot{q}^{+}-h\right)_{n}, \dot{\eta}_{\dagger}^{\diamond}\right\rangle \leq V^{\uparrow}\left(q_{n}, \dot{\eta}_{\dagger}^{\diamond}\right) ; \quad q_{n} \in C, \quad \text { for all } \dot{\eta}_{\dagger}^{\diamond}
$$

Together with (5.18) we obtain for $n \rightarrow \infty$,

$$
-\left\langle\left(M \ddot{q}^{+}-h\right)_{\dagger}, \dot{\eta}_{\dagger}^{\diamond}\right\rangle \leq \limsup _{n \rightarrow \infty} V^{\uparrow}\left(q_{n}, \dot{\eta}_{\dagger}^{\diamond}\right) \leq \Phi^{\dagger}\left(\dot{q}_{\dagger}^{+}, \dot{\eta}_{\dagger}^{\diamond}\right) \leq V^{\uparrow}\left(q_{\dagger}, \dot{\eta}_{\dagger}^{\diamond}\right) \quad \text { for all } \dot{\eta}_{\dagger}^{\diamond},
$$

where $q_{\dagger} \in C$ and $\dot{q}_{+}^{+} \in T_{C}\left(q_{\dagger}\right)$ due to Proposition 5.1. Suppose that Jourdain's principle holds at $t_{+}$, i.e. $-\left\langle\left(M \ddot{q}^{+}-h\right)_{\dagger}, \dot{\eta}_{\dagger}^{\diamond}\right\rangle \leq \Phi^{\uparrow}\left(\dot{q}_{\dagger}^{+}, \dot{\eta}_{\dagger}^{\diamond}\right)$. Due to $\Phi^{\uparrow}\left(\dot{q}_{\dagger}^{+}, \dot{\eta}_{\dagger}^{\diamond}\right) \leq V^{\uparrow}\left(q_{\dagger}, \dot{\eta}_{\dagger}^{\diamond}\right)$ the inequality $-\left\langle\left(M \ddot{q}^{+}-h\right)_{\dagger}, \dot{\eta}_{\dagger}^{\diamond}\right\rangle \leq V^{\uparrow}\left(q_{\dagger}, \dot{\eta}_{\dagger}^{\diamond}\right)$ also holds which is d'Alembert's principle at $t_{\dagger}$. Suppose that d'Alembert's principle holds for every $t_{n}>t_{\dagger}$. It holds particularly for the limit $t_{n} \rightarrow t_{\dagger}$, where $-\left\langle\left(M \ddot{q}^{+}-h\right)_{\dagger}, \dot{\eta}_{\dagger}^{\diamond}\right\rangle \leq \limsup _{n \rightarrow \infty} V^{\uparrow}\left(q_{n}, \dot{\eta}_{\dagger}^{\diamond}\right)$. Since $\limsup _{n \rightarrow \infty} V^{\uparrow}\left(q_{n}, \dot{\eta}_{\dagger}^{\diamond}\right) \leq \Phi^{\uparrow}\left(\dot{q}_{\dagger}^{+}, \dot{\eta}_{\dagger}^{\diamond}\right)$, we have $-\left\langle\left(M \ddot{q}^{+}-h\right)_{\dagger}, \dot{\eta}_{\dagger}^{\diamond}\right\rangle \leq \Phi^{\dagger}\left(\dot{q}_{\dagger}^{+}, \dot{\eta}_{\dagger}^{\diamond}\right)$ which is Jourdain's principle at $t_{\dagger}$. This proves Theorem 5.2 because $t_{\dagger} \in\left[t_{\mathrm{A}}, t_{\mathrm{E}}\right]$ is arbitrary. 
In the remaining part of the section we will sketch the connection between Jourdain's and Gauss' principles. The calculations are basically the same as in the first part and thus shortened. As in (5.1) we will consider, instead of the velocity potential $\tilde{\Phi}$, its first order approximation

$$
\bar{\Phi}\left(\dot{q}^{+}\right)=\tilde{\Phi}\left(\dot{q}_{\dagger}^{+}\right)+\tilde{\Phi}^{\uparrow}\left(\dot{q}_{\dagger}^{+}, \dot{q}^{+}-\dot{q}_{\dagger}^{+}\right) ; \quad \operatorname{dom} \bar{\Phi}=\dot{q}_{\dagger}^{+}+T_{T_{C}\left(q_{\dagger}\right)}\left(\dot{q}_{\dagger}^{+}\right),
$$

which is a l.s.c. proper convex function of $\dot{q}^{+}$, and which is independent of $q$. Additionally to the assumptions made in Proposition 5.1 we have

Proposition 5.3: Let $\bar{\Phi}: \mathbb{R}^{f} \rightarrow(-\infty,+\infty]$ be the l.s.c. proper convex function (5.21) with $\dot{q}_{\dagger}^{+} \in T_{C}\left(q_{\dagger}\right)$. Let $\left\{t_{n}\right\}$ be a sequence such that $t_{1}>t_{2}>\ldots>t_{\dagger}$, and $t_{n} \rightarrow t_{\dagger}$ for $n \rightarrow \infty$. Let $\dot{q}^{+}(t)$ be locally absolutely continuous such that $\dot{q}^{+}\left(t_{\dagger}\right)=\dot{q}_{\dagger}^{+}, \dot{q}^{+}\left(t_{n}\right)=\dot{q}_{n}^{+}$with $\dot{q}_{n}^{+} \in \operatorname{dom} \bar{\Phi}$ and $\dot{q}_{n}^{+}$not necessarily distinct from $\dot{q}_{\dagger}^{+}$. Then

$$
\begin{array}{lll}
\text { i) } & \bar{\Psi}^{\uparrow}\left(\ddot{q}_{\dagger}^{+}, \ddot{\eta}_{\dagger}^{\times}\right) \leq \bar{\Phi}^{\uparrow}\left(\dot{q}_{\dagger}^{+}, \ddot{\eta}_{\dagger}^{\times}\right) & \text {for all } \ddot{\eta}_{\dagger}^{\times}, \\
\text {ii) } & \limsup _{n \rightarrow \infty} \bar{\Phi}^{\uparrow}\left(\dot{q}_{n}^{+}, \ddot{\eta}_{\dagger}^{\times}\right) \leq \bar{\Phi}^{\uparrow}\left(\dot{q}_{\dagger}^{+}, \ddot{\eta}_{\dagger}^{\times}\right) & \text {for all } \ddot{\eta}_{\dagger}^{\times}, \\
\text {iii) } & \limsup _{n \rightarrow \infty} \bar{\Phi}^{\uparrow}\left(\dot{q}_{n}^{+}, \ddot{\eta}_{\dagger}^{\times}\right) \leq \bar{\Psi}^{\uparrow}\left(\ddot{q}_{\dagger}^{+}, \ddot{\eta}_{\dagger}^{\times}\right) & \text {for all } \ddot{\eta}_{\dagger}^{\times},
\end{array}
$$

where $\dot{q}_{n}^{+} \rightarrow \dot{q}_{\dagger}^{+}, \ddot{q}_{n}^{+} \rightarrow \ddot{q}_{\dagger}^{+}$as $n \rightarrow \infty, \bar{\Psi}(\cdot)=\bar{\Phi}^{\uparrow}\left(\dot{q}_{\dagger}^{+}, \cdot\right)$ is the acceleration potential (4.9) of $\bar{\Phi}$ at $\dot{q}_{\dagger}^{+}$, and $\ddot{q}_{\dagger}^{+}$is the right derivative of $\dot{q}^{+}$at time $t_{\dagger}$, i.e. the right acceleration of $q$ at time $t_{\dagger}$ with $\ddot{q}_{\dagger}^{+} \in T_{T_{C}\left(q_{\dagger}\right)}\left(\dot{q}_{\dagger}^{+}\right)$.

Proof: The proof of the first and the second assertion works analogously to the proof of Proposition 5.1. Since $\bar{\Psi}$ is a positively homogeneous convex function and hence subadditive we can apply (5.3)-(5.5) when replacing $\tilde{\Phi}, \dot{q}_{+}^{+}$, $\dot{\eta}_{\dagger}^{\diamond}$ by $\bar{\Psi}, \ddot{q}_{\dagger}^{+}, \ddot{\eta}_{\dagger}^{\times}$. Furthermore, $\bar{\Phi}(\cdot)$ is a proper convex function on $\mathbb{R}^{f}$, and $\dot{q}_{n}^{+} \in \operatorname{dom} \bar{\Phi}$ which is a closed convex set. Hence, the function $\bar{\Phi}^{\uparrow}\left(\cdot, \ddot{\eta}_{\dagger}^{\times}\right)$is u.s.c. In order to prove the third assertion we state, similarly to equation (5.6), the velocity potential $\bar{\Phi}$ at time $t_{n}$ with respect to the velocities $\dot{q}_{n}^{+}=\dot{q}^{+}\left(t_{n}\right)$ and $\dot{q}_{n}^{\diamond}=\dot{q}^{\diamond}\left(t_{n}\right)$. Since $\overline{\boldsymbol{\Phi}}$ is convex we have

$$
\bar{\Phi}^{\uparrow}\left(\dot{q}_{n}^{+}, \dot{q}_{n}^{\diamond}-\dot{q}_{n}^{+}\right) \leq \bar{\Phi}\left(\dot{q}_{n}^{\diamond}\right)-\bar{\Phi}\left(\dot{q}_{n}^{+}\right) .
$$

This equation holds for any arbitrary sequence $\left\{\dot{q}_{n}^{\diamond}\right\}$. It holds particularly for sequences $\left\{\dot{q}_{n}^{\times}\right\} \subset\left\{\dot{q}_{n}^{\diamond}\right\}$ approaching $\dot{q}_{\dagger}^{+}$for $n \rightarrow \infty$. Similarly to $(5.7)$ we set

$$
\dot{q}_{n}^{+}-\dot{q}_{\dagger}^{+}=\tau_{n} \ddot{q}_{n}^{+}, \quad \dot{q}_{n}^{\times}-\dot{q}_{\dagger}^{+}=\tau_{n} \ddot{q}_{n}^{\times},
$$

where $\tau_{n}=t_{n}-t_{\dagger}>0$, and $\tau_{n} \downarrow 0, \dot{q}_{n}^{+} \rightarrow \dot{q}_{\dagger}^{+}, \dot{q}_{n}^{\times} \rightarrow \dot{q}_{\dagger}^{+}$for $n \rightarrow \infty$. As in (5.8) we have

$$
\lim _{n \rightarrow \infty} \frac{1}{\tau_{n}}\left(\dot{q}_{n}^{+}-\dot{q}_{\dagger}^{+}\right)=\ddot{q}_{\dagger}^{+}, \quad \lim _{n \rightarrow \infty} \frac{1}{\tau_{n}}\left(\dot{q}_{n}^{\times}-\dot{q}_{\dagger}^{+}\right)=\ddot{q}_{\dagger}^{\times},
$$

which is the definition of the right accelerations of $\dot{q}^{+}(t)$ and $\dot{q}^{\times}(t)$ at time $t_{\dagger}$. Note that $\dot{q}^{\times}(t)$ has been chosen such that $\dot{q}^{\times}\left(t_{\dagger}\right)=\dot{q}^{+}\left(t_{\dagger}\right)$, but $\ddot{q}_{\dagger}^{\times}$is arbitrary which agrees with the classical approach that velocities (and displacements) must not be varied under acceleration variations. Since $\dot{q}_{n}^{+} \in \operatorname{dom} \bar{\Phi}=\dot{q}_{\dagger}^{+}+T_{T_{C}\left(q_{\dagger}\right)}\left(\dot{q}_{\dagger}^{+}\right)$, and $\dot{q}_{n}^{+}-\dot{q}_{\dagger}^{+}=\tau_{n} \ddot{q}_{n}^{+}$, and $\tau_{n}>0$, we have $\ddot{q}_{n}^{+} \in T_{T_{C}\left(q_{\dagger}\right)}\left(\dot{q}_{\dagger}^{+}\right)$for every $n$, particularly, $\ddot{q}_{\dagger}^{+} \in T_{T_{C}\left(q_{\dagger}\right)}\left(\dot{q}_{\dagger}^{+}\right)$because $\ddot{q}_{n}^{+} \rightarrow \ddot{q}_{\dagger}^{+}$when $n \rightarrow \infty$. Now we substitute (5.24) into (5.23) with $\left\{\dot{q}_{n}^{\times}\right\} \subset\left\{\dot{q}_{n}^{\diamond}\right\}$ and perform the same steps as in equations (5.9)-(5.12) in order to obtain

$$
\bar{\Phi}^{\uparrow}\left(\dot{q}_{n}^{+}, \ddot{q}_{n}^{\times}-\ddot{q}_{n}^{+}\right) \leq \bar{\Psi}\left(\ddot{q}_{n}^{\times}\right)-\bar{\Psi}\left(\ddot{q}_{n}^{+}\right),
$$

which holds for every sequence $\left\{\ddot{q}_{n}^{\times}\right\}$. As in (5.13) we choose sequences

$$
\ddot{q}_{n}^{\times}-\ddot{q}_{n}^{+}=\mu \ddot{\eta}_{\dagger k}^{\times}, \quad \mu>0,
$$

with arbitrary $\ddot{\eta}_{\dagger k}^{\times}, \ddot{\eta}_{\dagger}^{\times}$such that $\ddot{\eta}_{\dagger k}^{\times} \rightarrow \ddot{\eta}_{\dagger}^{\times}$when $k \rightarrow \infty$. With the help of (5.27) we eliminate $\ddot{q}_{n}^{\times}$from (5.26), take the "inf" with respect to $\mu$, let $k \rightarrow \infty$ and $n \rightarrow \infty$ as in (5.14)-(5.17). This finally provides

$$
\limsup _{n \rightarrow \infty} \bar{\Phi}^{\uparrow}\left(\dot{q}_{n}^{+}, \ddot{\eta}_{\dagger}^{\times}\right) \leq \bar{\Psi}^{\uparrow}\left(\ddot{q}_{\dagger}^{+}, \ddot{\eta}_{\dagger}^{\times}\right) \quad \text { for all } \ddot{\eta}_{\dagger}^{\times}
$$

and proves the third assertion of Proposition 5.3. $\|$

We expect even here that Proposition 5.3 may be generalized to certain classes of functions $\Phi\left(\dot{q}^{+}\right)=V^{\uparrow}\left(q, \dot{q}^{+}\right)$. In this case we may write according to $(5.22)$,

$$
\limsup _{n \rightarrow \infty} \Phi^{\uparrow}\left(\dot{q}_{n}^{+}, \ddot{\eta}_{\dagger}^{\times}\right) \leq \Psi^{\uparrow}\left(\ddot{q}_{\dagger}^{+}, \ddot{\eta}_{\dagger}^{\times}\right) \leq \Phi^{\uparrow}\left(\dot{q}_{\dagger}^{+}, \ddot{\eta}_{\dagger}^{\times}\right) \quad \text { for all } \ddot{\eta}_{\dagger}^{\times}
$$

Theorem 5.4: The principles of Jourdain (4.7) and Gauss (4.10) are equivalent if equation (5.29) holds. Suppose that $q(t)$ is given on the time interval $\left[t_{\mathrm{A}}, t_{\mathrm{E}}\right]$ such that Jourdain's principle holds for every $t_{\dagger} \in\left[t_{\mathrm{A}}, t_{\mathrm{E}}\right]$. Then Gauss' principle holds on $\left[t_{\mathrm{A}}, t_{\mathrm{E}}\right)$. If Gauss' principle holds on $\left[t_{\mathrm{A}}, t_{\mathrm{E}}\right]$, then Jourdain's principle also does. 
Proof: We state Jourdain's principle (4.7) at time $t_{n}>t_{\dagger}$ with $\left\{\dot{q}_{n}^{\times}\right\} \subset\left\{\dot{q}_{n}^{\diamond}\right\}$ and $\dot{q}_{n}^{\times}-\dot{q}_{n}^{+}=\tau_{n} \mu \ddot{\eta}_{\dagger}^{\times}$according to (5.24) and (5.27). For $n \rightarrow \infty$ we obtain together with (5.29),

$$
-\left\langle\left(M \ddot{q}^{+}-h\right)_{\dagger}, \ddot{\eta}_{\dagger}^{\times}\right\rangle \leq \limsup _{n \rightarrow \infty} \Phi^{\uparrow}\left(\dot{q}_{n}^{+}, \ddot{\eta}_{\dagger}^{\times}\right) \leq \Psi^{\uparrow}\left(\ddot{q}_{\dagger}^{+}, \ddot{\eta}_{\dagger}^{\times}\right) \leq \Phi^{\uparrow}\left(\dot{q}_{\dagger}^{+}, \ddot{\eta}_{\dagger}^{\times}\right) \quad \text { for all } \ddot{\eta}_{\dagger}^{\times},
$$

with $\dot{q}_{\dagger}^{+} \in T_{C}\left(q_{\dagger}\right)$ and $\ddot{q}_{\dagger}^{+} \in T_{T_{C}\left(q_{\dagger}\right)}\left(\dot{q}_{\dagger}^{+}\right)$. The rest of the proof works analogously to the proof of Theorem 5.2. $\|$

\section{Remarks}

The main result of Section 5 may be summarized as follows: The principles of d'Alembert, Jourdain, and Gauss are equivalent for a certain class of non-convex potential functions which are "not too far away" from the convex case. One important property of such systems may be expressed via the inequalities (5.20) and (5.30),

$$
-\left\langle M \ddot{q}^{+}-h, \ddot{\eta}^{\times}\right\rangle \leq \Psi^{\uparrow}\left(\ddot{q}^{+}, \ddot{\eta}^{\times}\right) \leq \Phi^{\uparrow}\left(\dot{q}^{+}, \ddot{\eta}^{\times}\right) \leq V^{\uparrow}\left(q, \ddot{\eta}^{\times}\right) \quad \text { for all } \ddot{\eta}^{\times},
$$

or, by taking into account the representation (2.8) of the generalized gradients (and subdifferentials) together with equation (6.1), via the inclusions

$$
-\left(M \ddot{q}^{+}-h\right) \in \partial \Psi\left(\ddot{q}^{+}\right) \subseteq \partial \Phi\left(\dot{q}^{+}\right) \subseteq \bar{\partial} V(q),
$$

cf. equations (4.3), (4.8), and (4.11). The advantages of introducing acceleration potentials and solving Gauss' principle instead of d'Alembert's principle are multiple: First of all we were able to proceed from a non-convex displacement potential to a convex and hence much better behaved acceleration potential which is positively homogeneous in addition. Secondly, since the set $\partial \Psi\left(\ddot{q}^{+}\right)$is contained in $\bar{\partial} V(q)$ (see $(6.2)$ ) the numerical evaluation of it becomes easier. Sometimes it may happen that $\partial \Psi\left(\ddot{q}^{+}\right)$consists of one element only whereas $\bar{\partial} V(q)$ is really "big". For systems allowing the formulation of the generalized gradients via linear complementarity conditions the dimension of the matrix of the corresponding Linear Complementarity Problem is directly related to the dimensions of the sets in (6.2). Finally, by the Principle of Gauss we achieved a representation of the dynamics equations which enables a direct access to the right accelerations of the system. Moreover, these accelerations are uniquely determined by the corresponding strictly convex optimization problem (4.13) which is the Principle of Least Constraints.

An interesting task is to examine the conditions under which the equations (6.1) and (6.2) can be written as equalities. Besides of the smooth case which is discussed in Section 7 we may derive some results when the velocities and/or accelerations vanish. First we will show that $V^{\uparrow}\left(q, \dot{\eta}^{\diamond}\right)=\Phi^{\uparrow}\left(0, \dot{\eta}^{\diamond}\right)$. For that we look again at equation (5.18) together with the sequence $\left\{q_{n}\right\}$ stated in equation (5.7), i.e. $q_{n}-q_{\dagger}=\tau_{n} \dot{q}_{n}^{+}$. Equation (5.18) holds for every sequence $\left\{q_{n}\right\}$. We consider a sequence such that $q_{1}=q_{2}=\ldots=q_{\dagger}$. Since $\tau_{n}>0$ we have $\dot{q}_{1}^{+}=\dot{q}_{2}^{+}=\ldots=\dot{q}_{\dagger}^{+}=0$. With this sequence equation $(5.18)$ becomes $V^{\uparrow}\left(q_{\dagger}, \dot{\eta}_{\dagger}^{\diamond}\right) \leq \Phi^{\uparrow}\left(0, \dot{\eta}_{\dagger}^{\diamond}\right) \leq V^{\uparrow}\left(q_{\dagger}, \dot{\eta}_{\dagger}^{\diamond}\right)$ which proves the equality $V^{\uparrow}\left(q_{\dagger}, \dot{\eta}_{\dagger}^{\diamond}\right)=\Phi^{\uparrow}\left(0, \dot{\eta}_{\dagger}^{\diamond}\right)$. The same can be done for sequences $\left\{\dot{q}_{n}^{+}\right\}$from $(5.24), \dot{q}_{n}^{+}-\dot{q}_{\dagger}^{+}=\tau_{n} \ddot{q}_{n}^{+}$. Choosing $\dot{q}_{1}^{+}=\dot{q}_{2}^{+}=\ldots=\dot{q}_{\dagger}^{+}$we have $\ddot{q}_{1}^{+}=\ddot{q}_{2}^{+}=\ldots=\ddot{q}_{\dagger}^{+}=0$ which yields together with (5.29) the equality $\Phi^{\uparrow}\left(\dot{q}_{\dagger}^{+}, \ddot{\eta}_{\dagger}^{\times}\right)=\Psi^{\uparrow}\left(0, \ddot{\eta}_{\dagger}^{\times}\right)$. Thus

$$
\Phi^{\uparrow}\left(0, \dot{\eta}^{\diamond}\right)=V^{\uparrow}\left(q, \dot{\eta}^{\diamond}\right) \quad \text { for all } \dot{\eta}^{\diamond}, \quad \text { and } \quad \Psi^{\uparrow}\left(0, \ddot{\eta}^{\times}\right)=\Phi^{\uparrow}\left(\dot{q}^{+}, \ddot{\eta}^{\times}\right) \quad \text { for all } \ddot{\eta}^{\times},
$$

and we have for the corresponding generalized gradients the well-known relations

$$
\partial \Phi(0)=\bar{\partial} V(q) \quad \text { and } \quad \partial \Psi(0)=\partial \Phi\left(\dot{q}^{+}\right) .
$$

If somebody is interested, for example, in a steady state motion, i.e. a motion with $\ddot{q}^{+}=0$, one obtains from (6.2) the implicit differential inclusion

$$
h\left(q, \dot{q}^{+}\right) \in \partial \Phi\left(\dot{q}^{+}\right) \subseteq \bar{\partial} V(q),
$$

where $\partial \Psi(0)=\partial \Phi\left(\dot{q}^{+}\right)$due to equation (6.4). Analogously we get from (6.2) the condition for equilibrium points $\left(\ddot{q}^{+}=0, \dot{q}^{+}=0\right)$ of the system,

$$
0 \in \bar{\partial} V(q)
$$

where $h(0,0)=0$ due to equations (3.6), (3.7), and $\partial \Psi(0)=\partial \Phi(0)=\bar{\partial} V(q)$.

In the following we will look a bit closer at the three different sets $\bar{\partial} V(q), \partial \Phi\left(\dot{q}^{+}\right)$, and $\partial \Psi\left(\ddot{q}^{+}\right)$in order to get a better understanding of their structure. By equation (4.2) these sets consist of forces which we will denote by $f_{V}^{+}, f_{\Phi}^{+}$, and $f_{\Psi}^{+}$, respectively. In a first step we will examine the connection between $\bar{\partial} V(q)$ and $\partial \Phi\left(\dot{q}^{+}\right)$. By equations (4.6) and (2.7) the velocity potential $\Phi\left(\dot{q}^{+}\right)$is defined to be

$$
\Phi\left(\dot{q}^{+}\right)=V^{\uparrow}\left(q, \dot{q}^{+}\right)=\sup \left\{\left\langle\dot{q}^{+},-f_{V}^{+}\right\rangle \mid-f_{V}^{+} \in \bar{\partial} V(q)\right\} .
$$

In terms of convex analysis [20] the velocity potential $\Phi\left(\dot{q}^{+}\right)$is the support function of the convex set $\bar{\partial} V(q)$, i.e. the conjugate of the indicator function of $\bar{\partial} V(q)$. From (6.7) we see immediately that

$$
\Phi\left(\dot{q}^{+}\right) \geq\left\langle\dot{q}^{+},-f_{V}^{+}\right\rangle \quad \text { for all }-f_{V}^{+} \in \bar{\partial} V(q)
$$


for fixed $\dot{q}^{+}$, which is Fenchel's inequality for indicator and support functions. Furthermore, it is known [20] that, for fixed $\dot{q}^{+}$, equation (6.8) holds as an equality if and only if we can find an element $-f_{\Phi}^{+}$of $\bar{\partial} V(q)$ which is a subgradient of $\Phi$ at $\dot{q}^{+}$, i.e.

$$
\Phi\left(\dot{q}^{+}\right)=\left\langle\dot{q}^{+},-f_{\Phi}^{+}\right\rangle \Leftrightarrow-f_{\Phi}^{+} \in \partial \Phi\left(\dot{q}^{+}\right) .
$$

Combining equations (6.7) and (6.9) we are able to state the subdifferential $\partial \Phi\left(\dot{q}^{+}\right)$as

$$
\partial \Phi\left(\dot{q}^{+}\right)=\left\{-f_{\Phi}^{+} \in \bar{\partial} V(q) \mid\left\langle\dot{q}^{+},-f_{\Phi}^{+}\right\rangle=\sup \left\langle\dot{q}^{+},-f_{V}^{+}\right\rangle,-f_{V}^{+} \in \bar{\partial} V(q)\right\} .
$$

In the same manner we obtain the set $\partial \Psi\left(\ddot{q}^{+}\right)$, i.e.

$$
\partial \Psi\left(\ddot{q}^{+}\right)=\left\{-f_{\Psi}^{+} \in \partial \Phi\left(\dot{q}^{+}\right) \mid\left\langle\ddot{q}^{+},-f_{\Psi}^{+}\right\rangle=\sup \left\langle\ddot{q}^{+},-f_{\Phi}^{+}\right\rangle,-f_{\Phi}^{+} \in \partial \Phi\left(\dot{q}^{+}\right)\right\} .
$$

By equation (6.10) we have shown that the subdifferential of the velocity potential consists of the elements $-f_{\Phi}^{+} \in \bar{\partial} V(q)$ for which the expression $\left\langle\dot{q}^{+},-f_{\Phi}^{+}\right\rangle$achieves its maximum when $\dot{q}^{+}$is fixed. The value of this maximum is given by equation (6.9), i.e. $\left\langle\dot{q}^{+},-f_{\Phi}^{+}\right\rangle=\Phi\left(\dot{q}^{+}\right)=V^{\uparrow}\left(q, \dot{q}^{+}\right)$. Analogously, the set $\partial \Psi\left(\ddot{q}^{+}\right)$consists of the elements $-f_{\Psi}^{+} \in \partial \Phi\left(\dot{q}^{+}\right)$for which the equality $\Phi^{\uparrow}\left(\dot{q}^{+}, \ddot{q}^{+}\right)=\left\langle\ddot{q}^{+},-f_{\Psi}^{+}\right\rangle$holds.

For completeness we will shortly discuss the case when we are interested in the accelerations from the past $\ddot{q}^{-}$. The equations of motion and the force law (cf. equations (3.21) and (4.1), (4.2)) are

$$
M \ddot{q}^{-}-h=f^{-}, \quad-f^{-} \in \bar{\partial} V(q) .
$$

According to equations (4.5), (4.7), and (4.10) we may state the three principles in the form

$$
\begin{array}{lll}
\text { (A) } & -\left\langle M \ddot{q}^{-}-h, q^{\star}-q\right\rangle \leq V^{\uparrow}\left(q, q^{\star}-q\right) ; & \text { for all } q^{\star}, q \in C, \\
\text { (J) } & -\left\langle M \ddot{q}^{-}-h, \dot{q}^{\diamond}-\dot{q}^{-}\right\rangle \leq \Phi^{\uparrow}\left(-\dot{q}^{-}, \dot{q}^{\diamond}-\dot{q}^{-}\right) ; & \text {for all } \dot{q}^{\diamond}, q \in C,-\dot{q}^{-} \in T_{C}(q), \\
\text { (G) } & -\left\langle M \ddot{q}^{-}-h, \ddot{q}^{\times}-\ddot{q}^{-}\right\rangle \leq \Psi^{\uparrow}\left(\ddot{q}^{-}, \ddot{q}^{\times}-\ddot{q}^{+}\right) ; & \text {for all } \ddot{q}^{\times}, q \in C,-\dot{q}^{-} \in T_{C}(q),-\ddot{q}^{-} \in T_{T_{C}(q)}\left(-\dot{q}^{-}\right),
\end{array}
$$

where, in correspondence with equations (4.6) and (4.9), the velocity and the acceleration potential are defined to be

$$
\Phi(p)=V^{\uparrow}(q, p), \quad \Psi(p)=\Phi^{\uparrow}\left(-\dot{q}^{-}, p\right) .
$$

As in (4.3), (4.8), and (4.11) we finally state the differential inclusions which are equivalent formulations of the three principles $(6.13)$,

$$
-\left(M \ddot{q}^{-}-h\right) \in \bar{\partial} V(q), \quad-\left(M \ddot{q}^{-}-h\right) \in \partial \Phi\left(-\dot{q}^{-}\right), \quad-\left(M \ddot{q}^{-}-h\right) \in \partial \Psi\left(\ddot{q}^{-}\right) .
$$

Note the additional negative sign in Jourdain's principle. Instead of $\Phi^{\uparrow}\left(\dot{q}^{+}, \dot{q}^{\diamond}-\dot{q}^{+}\right)$when dealing with the evolution of the system with respect to the future (4.7) we now have $\Phi^{\uparrow}\left(-\dot{q}^{-}, \dot{q}^{\diamond}-\dot{q}^{-}\right)$, see second equation of (6.13). This sign enters the problem at equations (5.10) and (5.11), because now we must consider a sequence of time points $\left\{t_{n}\right\}$ such that $t_{1}<t_{2}<\ldots<t_{\dagger}$, hence $\tau_{n}=t_{n}-t_{\dagger}<0$. When eliminating $\tau_{n}$ from (5.10) we obtain now, instead of (5.11), the inequality

$$
\tilde{V}^{\uparrow}\left(q_{n},-\left(\dot{q}_{n}^{\diamond}-\dot{q}_{n}^{+}\right)\right) \leq V^{\uparrow}\left(q_{\dagger},-\dot{q}_{n}^{\diamond}\right)-V^{\uparrow}\left(q_{\dagger},-\dot{q}_{n}^{+}\right) .
$$

This negative sign will drop out at the acceleration level in the same manner as it appeared at the velocity level. Thus we are still able to formulate Gauss' principle in terms of a strictly convex optimization problem (4.13) and to obtain uniquely determined accelerations $\ddot{q}^{-}$.

\section{Example: Smooth potential functions}

In this section we will discuss the classical case of smooth potential functions. We assume that $V(q)$ is a $C^{1}$-potential with dom $V=\mathbb{R}^{f}$, i.e. $V(q)$ is continuously differentiable on $\mathbb{R}^{f}$. Potentials like that contain all classical displacement interaction forces such as forces resulting from gravity, forces from springs with linear or smooth nonlinear force characteristics, or even springs the force characteristics of which are continuous but contain corners.

Throughout this section we cancel the upper indices "+" and "-" because there is no reason for a jump in the velocities or in the accelerations as we will see. Since $V(q)$ is differentiable the generalized gradients and generalized directional derivatives may be replaced by the gradients and the corresponding inner products as it was already pointed out in equation (2.18). Particularly, the velocity and the acceleration potential in equations (4.6) and (4.9) become

$$
\Phi(p)=\langle\nabla V(q), p\rangle, \quad \Psi(p)=\langle\nabla \Phi(\dot{q}), p\rangle=\langle\nabla V(q), p\rangle .
$$

With it, we may obtain the generalized gradients and subdifferentials in (6.2). They consist of one element only,

$$
\bar{\partial} V(q)=\partial \Phi(\dot{q})=\partial \Psi(\ddot{q})=\nabla V(q),
$$


and the three corresponding differential inclusions (4.3), (4.8), (4.11) reduce to a single differential equation well known from classical mechanics,

$$
-(M \ddot{q}-h)=\nabla V(q) .
$$

This equation, multiplied by the displacement-, velocity-, and acceleration-variations yields the principles (4.5), (4.7), and (4.10). They hold as equalities, i.e.

$$
\begin{aligned}
& \text { (A) } \quad-\left\langle M \ddot{q}-h, q^{\star}-q\right\rangle=\left\langle\nabla V(q), q^{\star}-q\right\rangle ; \quad \text { for all } q^{\star} \text {, } \\
& \text { (J) } \quad-\left\langle M \ddot{q}-h, \dot{q}^{\diamond}-\dot{q}\right\rangle=\left\langle\nabla V(q), \dot{q}^{\diamond}-\dot{q}\right\rangle ; \quad \text { for all } \dot{q}^{\diamond} \text {, } \\
& \text { (G) } \quad-\left\langle M \ddot{q}-h, \ddot{q}^{\times}-\ddot{q}\right\rangle=\left\langle\nabla V(q), \ddot{q}^{\times}-\ddot{q}\right\rangle ; \text { for all } \ddot{q}^{\times},
\end{aligned}
$$

which can also be seen directly from (4.5) by choosing variations $\left(q^{\star}-q\right)$ and $-\left(q^{\star}-q\right)$, and analogously from (4.7), and (4.10) concerning appropriate variations. Finally, the Principle of Least Constraints (4.13) becomes

$$
\text { (Z) } \quad \ddot{q}=\arg \min \{f(\ddot{\bar{q}})\} ; \quad f(\ddot{q})=\frac{1}{2}\langle M \ddot{q}, \ddot{q}\rangle-\langle h, \ddot{q}\rangle+\langle\nabla V(q), \ddot{q}\rangle,
$$

which is a classical quadratic program without constraints.

\section{Example: Unilateral constraints}

It is a well known fact by now that unilateral constraints may be taken into account by indicator functions. We will consider the case of a multibody system, the displacements of which being subjected to some geometrical restrictions $q \in \operatorname{dom} V=C$. The indicator function $I_{C}$ of a set $C$ is defined by

$$
I_{C}(q)=\left\{\begin{array}{lll}
0 & \text { if } & q \in C \\
+\infty & \text { if } & q \notin C
\end{array},\right.
$$

and has some important connections to the normal and the tangential cone of $C$. In [21], [22] it is shown that

$$
\bar{\partial} I_{C}(q)=N_{C}(q)
$$

and

$$
I_{C}^{\uparrow}(q, p)=I_{T_{C}(q)}(p)
$$

We assume that only forces resulting from unilateral constraints act on the multibody system. In this case the displacement potential $V(q)$ is

$$
V(q)=I_{C}(q) .
$$

In order to derive the velocity potential (4.6) and the acceleration potential (4.9) we just have to apply equation (8.3). We obtain

$$
\Phi(p)=I_{C}^{\uparrow}(q, p)=I_{T_{C}(q)}(p), \quad \Psi(p)=I_{T_{C}(q)}^{\uparrow}\left(\dot{q}^{+}, p\right)=I_{T_{T_{C}(q)}\left(\dot{q}^{+}\right)}(p) .
$$

With the help of (8.2) we get immediately from (8.4) and (8.5) the three sets $\bar{\partial} V(q), \partial \Phi\left(\dot{q}^{+}\right)$, and $\partial \Psi\left(\ddot{q}^{+}\right)$. They are

$$
\bar{\partial} V(q)=N_{C}(q), \quad \partial \Phi\left(\dot{q}^{+}\right)=N_{T_{C}(q)}\left(\dot{q}^{+}\right), \quad \partial \Psi\left(\ddot{q}^{+}\right)=N_{T_{T_{C}(q)}\left(\dot{q}^{+}\right)}\left(\ddot{q}^{+}\right),
$$

and the differential inclusion (6.2) becomes

$$
-\left(M \ddot{q}^{+}-h\right) \in N_{T_{T_{C}(q)}\left(\dot{q}^{+}\right)}\left(\ddot{q}^{+}\right) \subseteq N_{T_{C}(q)}\left(\dot{q}^{+}\right) \subseteq N_{C}(q) .
$$

At that point, note the polarity (2.3) between the normal and the tangential cones. Due to (8.7) we obviously have

$$
T_{C}(q) \subseteq T_{T_{C}(q)}\left(\dot{q}^{+}\right) \subseteq T_{T_{T_{C}(q)}\left(\dot{q}^{+}\right)}\left(\ddot{q}^{+}\right) .
$$

The tangential cones increase from the displacement level to the acceleration level in the same manner as the corresponding normal cones decrease. One may say that the accelerations are in some sense less constrained than the displacements, a fact completely different from bilaterally constrained motion as we will see. Using the potential functions in $(8.4),(8.5)$, the three principles $(4.5),(4.7)$, and (4.10) become

$$
\begin{array}{lll}
\text { (A) } & -\left\langle M \ddot{q}^{+}-h, q^{\star}-q\right\rangle \leq I_{C}^{\uparrow}\left(q, q^{\star}-q\right) ; & \text { for all } q^{\star}, q \in C, \\
\text { (J) } & -\left\langle M \ddot{q}^{+}-h, \dot{q}^{\diamond}-\dot{q}^{+}\right\rangle \leq I_{T_{C}(q)}^{\uparrow}\left(\dot{q}^{+}, \dot{q}^{\diamond}-\dot{q}^{+}\right) ; & \text {for all } \dot{q}^{\diamond}, q \in C, \dot{q}^{+} \in T_{C}(q), \\
\text { (G) } & -\left\langle M \ddot{q}^{+}-h, \ddot{q}^{\times}-\ddot{q}^{+}\right\rangle \leq I_{T_{T_{C}(q)}^{\uparrow}\left(\dot{q}^{+}\right)}\left(\ddot{q}^{+}, \ddot{q}^{\times}-\ddot{q}^{+}\right) ; & \text {for all } \ddot{q}^{\times}, q \in C, \dot{q}^{+} \in T_{C}(q), \ddot{q}^{+} \in T_{T_{C}(q)}\left(\dot{q}^{+}\right) .
\end{array}
$$


We apply again equation (8.3) to the right-hand sides of the inequalities in (8.9) and choose the variations of the displacements, velocities, and accelerations such that they belong to the sets specified by the resulting indicator functions. The values of the indicators are then equal to zero, and (8.9) becomes

$$
\begin{array}{llll}
\text { (A) } & -\left\langle M \ddot{q}^{+}-h, q^{\star}-q\right\rangle \leq 0 ; & \text { for all }\left(q^{\star}-q\right) \in T_{C}(q), & q \in C, \\
\text { (J) } & -\left\langle M \ddot{q}^{+}-h, \dot{q}^{\diamond}-\dot{q}^{+}\right\rangle \leq 0 ; & \text { for all }\left(\dot{q}^{\diamond}-\dot{q}^{+}\right) \in T_{T_{C}(q)}\left(\dot{q}^{+}\right), & q \in C, \dot{q}^{+} \in T_{C}(q), \\
\text { (G) } & -\left\langle M \ddot{q}^{+}-h, \ddot{q}^{\times}-\ddot{q}^{+}\right\rangle \leq 0 ; & \text { for all }\left(\ddot{q}^{\times}-\ddot{q}^{+}\right) \in T_{T_{T_{C}(q)}\left(\dot{q}^{+}\right)}\left(\ddot{q}^{+}\right), & q \in C, \dot{q}^{+} \in T_{C}(q), \ddot{q}^{+} \in T_{T_{C}(q)}\left(\dot{q}^{+}\right) .
\end{array}
$$

Setting $M \ddot{q}^{+}-h=f^{+}$(cf. equation (4.1)) we see that the virtual work, power, ... produced by forces from unilateral constraints is always greater than or equal to zero. The Principle of d'Alembert for unilateral constraints was stated in 1821 by FourIER and can also be found in [26], [13]. MorEAU's work is completely based on Jourdain's principle in the second line of (8.10) but contains additionally impacts and Coulomb friction [8], [18]. The Principle of Gauss as stated in the third equation of (8.10) can be found in [13] and is often heuristically used in multibody dynamics, cf. for example [14]. Taking the acceleration potential (8.5) we may state the Principle of Least Constraints (4.13),

$$
\ddot{q}^{+}=\arg \min \left\{f\left(\ddot{\bar{q}}^{+}\right)\right\} ; \quad f\left(\ddot{q}^{+}\right)=\frac{1}{2}\left\langle M \ddot{q}^{+}, \ddot{q}^{+}\right\rangle-\left\langle h, \ddot{q}^{+}\right\rangle+I_{T_{T_{C}(q)}\left(\dot{q}^{+}\right)}\left(\ddot{q}^{+}\right),
$$

which is a strictly convex program with inequality constraints, i.e. find $\ddot{q}^{+}$such that $\frac{1}{2}\left\langle M \ddot{q}^{+}, \ddot{q}^{+}\right\rangle-\left\langle h, \ddot{q}^{+}\right\rangle$becomes minimal under the restriction that $\ddot{q}^{+}$belongs to the closed convex cone $T_{T_{C}(q)}\left(\dot{q}^{+}\right)$.

\section{Example: Bilateral constraints}

Bilateral constraints are completely included in the concept describing the unilateral case. In order to treat them we just have to find an appropriate set defining the admissible displacements, and then to apply the indicator function on it. We assume the system to be constrained on a $C^{1}$-manifold $M$. With that assumption we may cancel the upper indices "+" and "-" because systems like that are free of jumps in the velocities or in the accelerations. The displacement potential is then, according to equation (8.4),

$$
V(q)=I_{M}(q)
$$

where $I_{M}(q)$ is the indicator function of the manifold $M$. Since $M$ is $C^{1}$ it consists of regular points only, i.e. the tangent cone $T_{M}(q)$ and the contingent cone $K_{M}(q)$ of $M$ coincide for every point $q \in M$. Moreover, $T_{M}(q)$ reduces to the classical tangent space to $M$ at $q$ which is a subspace of $\mathbb{R}^{f}$. Particularly we have $-p \in T_{M}(q)$ if $p \in T_{M}(q)$. The normal cone $N_{M}(q)$ is also a subspace of $\mathbb{R}^{f}$. It is the orthogonal complement of $T_{M}(q)$ which can be seen immediately from equation (2.3),

$$
N_{M}(q)=\left\{-f \mid\langle-f, p\rangle=0 \text { for all } p \in T_{M}(q)\right\} .
$$

Since $-p \in T_{M}(q)$ if $p \in T_{M}(q)$ we have replaced the inequality in (2.3) by the corresponding equality $\langle-f, p\rangle=0$, i.e. the elements of $N_{M}(q)$ and $T_{M}(q)$ are orthogonal. Note especially that the tangent space to $M$ at $q \in M$ and the tangent space to $T_{M}(q)$ at $\dot{q} \in T_{M}(q)$ are identical. Thus, instead of the inclusion (8.8) we now have equality,

$$
T_{M}(q) \equiv T_{T_{M}(q)}(\dot{q}) \equiv T_{T_{T_{M}(q)}(\dot{q})}(\ddot{q})
$$

if $q \in M, \dot{q} \in T_{M}(q)$, and $\ddot{q} \in T_{T_{M}(q)}(\dot{q})$. The same holds for the normal spaces due to orthogonality, i.e.

$$
N_{T_{T_{M}(q)}(\dot{q})}(\ddot{q}) \equiv N_{T_{M}(q)}(\dot{q}) \equiv N_{M}(q) .
$$

With it, the differential inclusion (8.7) reduces to

$$
-(M \ddot{q}-h) \in N_{M}(q) \text {. }
$$

Since $M \ddot{q}-h=f$ we have $-f \in N_{M}(q)$, i.e. the classical result that constraint forces are orthogonal to the manifold $M$. They are additionally not restricted to any direction because $f$ also belongs to $N_{M}(q)$ if $-f$ does. With the help of (9.3) we rewrite $(8.10)$ and obtain

$$
\begin{array}{lll}
\text { (A) } & -\left\langle M \ddot{q}-h, q^{\star}-q\right\rangle=0 ; & \text { for all }\left(q^{\star}-q\right) \in T_{M}(q), q \in M, \\
\text { (J) } & -\left\langle M \ddot{q}-h, \dot{q}^{\diamond}-\dot{q}\right\rangle=0 ; & \text { for all }\left(\dot{q}^{\diamond}-\dot{q}\right) \in T_{M}(q), q \in M, \dot{q} \in T_{M}(q), \\
\text { (G) } & -\left\langle M \ddot{q}-h, \ddot{q}^{\times}-\ddot{q}\right\rangle=0 ; & \text { for all }\left(\ddot{q}^{\times}-\ddot{q}\right) \in T_{M}(q), q \in M, \dot{q} \in T_{M}(q), \ddot{q} \in T_{M}(q),
\end{array}
$$

which are the classical principles of d'Alembert, Jourdain, and Gauss. The equalities in (9.6) result from the property that $-\left(q^{\star}-q\right) \in T_{M}(q)$ if $\left(q^{\star}-q\right) \in T_{M}(q)$ and so on. In classical text books the terms $\left(q^{\star}-q\right),\left(\dot{q}^{\diamond}-\dot{q}\right)$, and $\left(\ddot{q}^{\times}-\ddot{q}\right)$ are usually denoted by $\delta q, \delta^{\prime} \dot{q}$, and $\delta^{\prime \prime} \ddot{q}$, and are called virtual displacements, virtual velocities, and virtual accelerations. Since $M \ddot{q}-h=f$ we see from (9.6) that constraint forces produce no virtual work, power, etc. Finally we state 
the classical Principle of Least Constraints (4.13)

$$
\text { (Z) } \quad \ddot{q}=\arg \min \{f(\ddot{\bar{q}})\} ; \quad f(\ddot{q})=\frac{1}{2}\langle M \ddot{q}, \ddot{q}\rangle-\langle h, \ddot{q}\rangle+I_{T_{M}(q)}(\ddot{q}),
$$

which is a strictly convex program with linear equality constraints, i.e. find $\ddot{q}$ such that $\frac{1}{2}\langle M \ddot{q}, \ddot{q}\rangle-\langle h, \ddot{q}\rangle$ becomes minimal under the restriction that $\ddot{q}$ belongs to the linear space $T_{M}(q)$. This relation may be found in many classical text books as well as in [27] where emphasis is put on the computer oriented formulation and evaluation of closed loop systems.

\section{Nonholonomic systems}

Up to now we have only considered potential functions $\Phi, \Psi$ which result from a displacement potential $V(q)$. With this approach one is able to take into account a vast class of displacement dependent interaction forces of single-valued and set-valued type as described in the foregoing sections. However, there are other types of forces depending also on velocities which share one common property: There is no way to express them via displacement potentials, i.e. they are non-integrable. It is well known that viscous damping belongs to this class. Other examples are every kind of dry friction (i.e. Coulomb friction with given normal force) as well as nonholonomic constraints. Surprisingly all forces mentioned above fit into the concept of using velocity potentials. As we have seen in Section 4 one is able to express displacement dependent forces also by velocity potentials $\Phi$. On the other hand, there are the viscous and the dry friction type forces which demand a representation via velocity potentials. Finally, by using indicator-like (velocity-) potential functions one is able to take into account even forces resulting from (non-)holonomic constraints. Thus we assume force laws of the form

$$
-f^{+} \in \bar{\partial} \Phi\left(\dot{q}^{+}\right)
$$

where $\bar{\partial} \Phi\left(\dot{q}^{+}\right)$denotes the generalized gradient of a velocity potential $\Phi\left(\dot{q}^{+}\right)$defined by

$$
\Phi\left(\dot{q}^{+}\right)=\Pi\left(q, \dot{q}^{+}\right)
$$

The properties of the function $\Pi: \mathbb{R}^{f} \times \mathbb{R}^{f} \rightarrow \mathbb{R} \cup\{+\infty\}$ that are required in order to be able to pass from Jourdain's to Gauss' principle and vice versa are not yet known. We expect, however, that $\Pi\left(q, \dot{q}^{+}\right)$should be at least u.s.c. with respect to $q$, l.s.c. with respect to $\dot{q}^{+}$, and the epigraph of $\Phi$ should consist of regular points only. Furthermore we assume that dom $\Pi=C \times S(q)$, where $S$ is a convex cone depending on the displacements $q$. For example, we have $\Pi\left(q, \dot{q}^{+}\right)=V^{\uparrow}\left(q, \dot{q}^{+}\right)$due to (4.6) with dom $\Pi=C \times T_{C}(q)$ when forces resulting from displacement potentials are considered. In this setting we may use Jourdain's and Gauss' principle as in equations (4.7) and (4.10),

$$
\begin{array}{ll}
-\left\langle M \ddot{q}^{+}-h, \dot{q}^{\diamond}-\dot{q}^{+}\right\rangle \leq \Phi^{\uparrow}\left(\dot{q}^{+}, \dot{q}^{\diamond}-\dot{q}^{+}\right) ; \quad \text { for all } \dot{q}^{\diamond}, q \in C, \dot{q}^{+} \in S(q), \\
-\left\langle M \ddot{q}^{+}-h, \ddot{q}^{\times}-\ddot{q}^{+}\right\rangle \leq \Psi^{\uparrow}\left(\ddot{q}^{+}, \ddot{q}^{\times}-\ddot{q}^{+}\right) ; \quad \text { for all } \ddot{q}^{\times}, q \in C, \dot{q}^{+} \in S(q), \ddot{q}^{+} \in T_{S(q)}\left(\dot{q}^{+}\right),
\end{array}
$$

where the acceleration potential $\Psi\left(\ddot{q}^{+}\right)$is the convex l.s.c. function defined in (4.9), and d'Alembert's principle does no longer exist due to the non-integrability of the velocity potential. The corresponding differential inclusions are (cf. equations (4.8) and (4.11)),

$$
-\left(M \ddot{q}^{+}-h\right) \in \bar{\partial} \Phi\left(\dot{q}^{+}\right), \quad-\left(M \ddot{q}^{+}-h\right) \in \partial \Psi\left(\ddot{q}^{+}\right) .
$$

Note that we have used the subdifferential instead of the generalized gradient in the second inclusion in (10.4) because $\Psi$ is a convex function. Finally we may state the Principle of Least Constraints as in (4.13),

$$
\ddot{q}^{+}=\arg \min \left\{f\left(\ddot{\bar{q}}^{+}\right)\right\} ; \quad f\left(\ddot{q}^{+}\right)=\frac{1}{2}\left\langle M \ddot{q}^{+}, \ddot{q}^{+}\right\rangle-\left\langle h, \ddot{q}^{+}\right\rangle+\Psi\left(\ddot{q}^{+}\right),
$$

which is obtained after "integrating" the second inclusion in (10.4). As in (4.13) we have a strictly convex cost function which leads to unique optimal solutions $\ddot{q}^{+}$.

For completeness we will also state the two principles when dealing with the accelerations from the past, cf. also equations $(6.12)-(6.15)$ for displacement potentials. We have

$$
\begin{array}{lll}
(\mathrm{J}) & -\left\langle M \ddot{q}^{-}-h, \dot{q}^{\diamond}-\dot{q}^{-}\right\rangle \leq \Phi^{\uparrow}\left(\dot{q}^{-}, \dot{q}^{\diamond}-\dot{q}^{-}\right) ; & \text {for all } \dot{q}^{\diamond}, q \in C, \dot{q}^{-} \in S(q), \\
(\mathrm{G}) & -\left\langle M \ddot{q}^{-}-h, \ddot{q}^{\times}-\ddot{q}^{-}\right\rangle \leq \Psi^{\uparrow}\left(-\ddot{q}^{-}, \ddot{q}^{\times}-\ddot{q}^{-}\right) ; & \text {for all } \ddot{q}^{\times}, q \in C, \dot{q}^{-} \in S(q),-\ddot{q}^{-} \in T_{S(q)}\left(\dot{q}^{-}\right),
\end{array}
$$

and the corresponding inclusions are

$$
-\left(M \ddot{q}^{-}-h\right) \in \bar{\partial} \Phi\left(\dot{q}^{-}\right), \quad-\left(M \ddot{q}^{-}-h\right) \in \partial \Psi\left(-\ddot{q}^{-}\right) .
$$

As in (6.13) and (6.15) an additional negative sign enters the equations, but now on the acceleration level. After "integrating" the second inclusion in (10.7) we obtain the cost function

$$
f\left(\ddot{q}^{-}\right)=\frac{1}{2}\left\langle M \ddot{q}^{-}, \ddot{q}^{-}\right\rangle-\left\langle h, \ddot{q}^{-}\right\rangle-\Psi\left(-\ddot{q}^{-}\right) .
$$


This is a non-convex function because $\frac{1}{2}\left\langle M \ddot{q}^{-}, \ddot{q}^{-}\right\rangle$is strictly convex and $-\Psi$ is concave. Thus the Principle of Least Constraints reduces to the problem: Find $\ddot{q}^{-}$such that the cost function $f$ becomes stationary. Uniqueness with respect to the accelerations $\ddot{q}^{-}$may no longer be expected which is a well-known property of dry friction: Nobody knows whether or when a body has stopped moving under the influence of dry friction if it is resting on a surface. Uniqueness holds for example if the positively homogeneous function $\Psi$ is additionally linear, i.e. in the case of viscous damping or nonholonomic linear constraints. Finally one should note that the source of the non-uniqueness of the accelerations from the past is closely related to the reintrant corner problem: Since $\Psi$ is a positively homogeneous convex function its epigraph is a convex cone. Consider now the concave function $-\Psi$. Its epigraph coincides with the contingent cone at $(0,-\Psi(0))$ which is generally a non-convex set. Thus $K_{\text {epi }-\Psi}(0,-\Psi(0)) \neq T_{\text {epi }-\Psi}(0,-\Psi(0))$, i.e. a reintrant corner.

\section{Conclusion}

The topic of the presented paper may be described as follows: When considering the velocities of a MBS as functions of bounded variation the accelerations of the system exist almost everywhere. In this case there must be a way to determine their values directly from the equations of motion. For that purpose we considered at first the equations of motion of a system under the influence of forces which were derived by generalized differentiation from a displacement potential. The corresponding differential inclusion was reformulated in terms of a hemivariational inequality; this is the Principle of d'Alembert. Following classical mechanics we also introduced a variational principle on the velocity and on the acceleration level, i.e. the Principles of Jourdain and Gauss, respectively. We expect the equivalence of the three principles for rather general classes of potential functions to hold. The main ideas of the proof were sketched in Section 5. We showed by examples that classical smooth interaction laws, unilateral constraints, and smooth bilateral constraints were covered by the chosen approach, where in the latter case the principles turned out to be the classical ones. In Section 10 this approach was extended to non-integrable potential functions on velocity level in order to take into account additional forces resulting from viscous damping, dry friction, and nonholonomic constraints. At a first view these forces seem to be of completely different nature sharing only the property of non-integrability of some related functions. It is a remarkable fact that all of them can be treated by the unified concept of using velocity potentials.

For complicated mechanical systems with given (displacement-) potential one should rather try to apply some decomposition rules than to derive the corresponding velocity and acceleration potential via the definition of the generalized directional derivative. The method of treating decomposed force interactions coincides also with the natural way of modelling mechanical systems: Starting from a physical model one develops an appropriate mathematical description of it. One step in this process consists of assigning to each single force in the model a reasonable force characteristic. At this point the computation of the corresponding parts of the overall displacement-, velocity-, and accelerationpotential is usually much easier than after summing up all the forces. This decomposition technique, applied to MBS depending also explicitely on time will be the subject of a forthcoming paper.

\section{Acknowledgements}

The author would like to thank Prof. P. D. PAnAgiotopoulos for his extremely helpful discussions, continuous encouragement and support. The author also wishes to express his thanks to Dr. Z. NANIEWICZ for many stimulating conversations and helpful suggestions during the preparation of the paper. The financial support of the Alexander von Humboldt Foundation is gratefully acknowledged.

\section{References}

1 Duvaut, G.; Lions, J. L.: Un problème d'élasticité avec frottement. J. de Mécanique 10 (1971), 409-420.

2 Panagiotopoulos, P. D.: Inequality Problems in Mechanics and Applications. Convex and Nonconvex Energy Functions. Birkhäuser Verlag, Basel 1985.

3 Panagiotopoulos, P. D.: Hemivariational Inequalities. Applications in Mechanics and Engineering. Springer Verlag, BerlinHeidelberg 1993.

4 Appell, P.: Traité de mécanique rationelle. Tome I: Statique-dynamique du point. Gauthier-Villars, Paris 1893.

5 Hamel, G.: Theoretische Mechanik. Springer Verlag, Berlin 1967.

6 Lanczos, C.: The Variational Principles of Mechanics. University of Toronto Press, Toronto 1966.

7 Delassus, E. : Mémoire sur la théorie des liaisons finies unilatérales. Ann. Sci. Ecole Norm. Sup. 34 (1917), 95-179.

8 Moreau, J. J.: Unilateral Contact and Dry Friction in Finite Freedom Dynamics. Non-Smooth Mechanics and Applications. CISM Courses and Lectures, Vol. 302, Springer Verlag, Wien 1988.

9 Moreau, J. J.: Les liaisons unilatérales et le Principe de Gauss. C.R. Acad. Sci. Paris 256 (1963), 871-874.

10 Panagiotopoulos, P. D.: Ungleichungsprobleme und Differentialinklusionen in der Analytischen Mechanik. Annual Public. School of Technology, Aristotle University Thessaloniki, Greece, Vol. @' (1982), 100-139.

11 Monteiro Marques, M. D. P.: Differential Inclusions in Nonsmooth Mechanical Problems. Shocks and Dry Friction. Birkhäuser Verlag, Basel 1993. 
12 Brogliato, B.: Nonsmooth Impact Mechanics. Models, Dynamics and Control. Lect. Notes in Control and Information Sciences Vol. 220, Springer Verlag, Heidelberg-New York 1996.

13 Glocker, Ch.: Dynamik von Starrkörpersystemen mit Reibung und Stößen. VDI-Fortschrittberichte Mechanik/Bruchmechanik, Reihe 18, Nr. 182, VDI-Verlag, Düsseldorf 1995.

14 Pfeiffer, F.; Glocker, Ch.: Multibody Dynamics with Unilateral Contacts. John Wiley \& Sons, New York 1996.

15 Pfeiffer, F.: Dynamical systems with time-varying or unsteady structure. ZAMM 71 (1991) 4, T6-T22.

16 Pang, J. S.; Trinkle, J. C.: Complementarity formulations and existence of solutions of multi-rigid-body contact problems with Coulomb friction. Math. Programming (to appear).

17 Lötstedt, P.: A numerical method for the simulation of mechanical systems with unilateral constraints. TRITA-NA-7920, Dept. of Numerical Analysis and Computing Science, The Royal Institute of Technology, Sweden 1979.

18 Moreau, J. J.: Bounded Variation in Time. Topics in Nonsmooth Mechanics, Birkhäuser Verlag, Basel 1988.

19 Aubin, J. P.; Ekeland, I.: Applied Nonlinear Analysis. Wiley-Inter-Science, New York 1984.

20 Rockafellar, R. T.: Convex Analysis. Princeton University Press, Princeton, New Jersey 1972.

21 Rockafellar, R. T.: La théorie des sous-gradients et ses applications à l'optimization. Fonctions convexes et non-convexes. Les Presses de l'Université de Montréal, Montréal 1979.

22 Rockafellar, R. T.: Generalized directional derivatives and subgradients of non-convex functions. Canadian J. Math. 32 (1980), 257-280

23 Rudin, W.: Real and Complex Analysis. Tata McGraw-Hill Publ., New Delhi 1981.

24 Ambrosio, L.: Existence theory for a new class of variational problems. Arch. Rat. Mech. Analysis 111 (1990), $291-322$.

25 Maisser, P.: Analytische Mechanik von Mehrkörpersystemen. ZAMM 68 (1988) 10, 463-481.

26 May, H.; Pangiotopoulos, P. D.: F. H. Clarke's generalized gradient and Fourier's principle. ZAMM 65 (1985) 2, 125-126.

27 Lilov, L.; Lorer, M. : Dynamic analysis of multirigid-body system based on the Gauss principle. ZAMM 62 (1982) 10, $539-545$.

Address: Dr. Christoph Glocker, Dept. of Civil Engineering, Aristotle University of Thessaloniki, GR-54006 Thessaloniki, Greece. On leave from the Lehrstuhl B für Mechanik, Technische Universität München, D-80290 München, Germany. 